ARTICLE

DOI: $10.1038 /$ s41467-018-07033-z

\title{
Neuronal SIRT1 regulates macronutrient-based diet selection through FGF21 and oxytocin signalling in mice
}

Sho Matsui ${ }^{1}$, Tsutomu Sasaki (i] 1, Daisuke Kohno1,2, Keisuke Yaku ${ }^{3,4}$, Ayumu Inutsuka (D) 5,6, Hiromi Yokota-Hashimoto ${ }^{1}$, Osamu Kikuchi ${ }^{1}$, Takayoshi Suga (1) ${ }^{1}$, Masaki Kobayashi ${ }^{1}$, Akihiro Yamanaka ${ }^{5}$, Akihiro Harada7 , Takashi Nakagawa (1) ${ }^{3,4}$, Tatsushi Onaka ${ }^{6}$ \& Tadahiro Kitamura ${ }^{1}$

Diet affects health through ingested calories and macronutrients, and macronutrient balance affects health span. The mechanisms regulating macronutrient-based diet choices are poorly understood. Previous studies had shown that NAD-dependent deacetylase sirtuin-1 (SIRT1) in part influences the health-promoting effects of caloric restriction by boosting fat use in peripheral tissues. Here, we show that neuronal SIRT1 shifts diet choice from sucrose to fat in mice, matching the peripheral metabolic shift. SIRT1-mediated suppression of simple sugar preference requires oxytocin signalling, and SIRT1 in oxytocin neurons drives this effect. The hepatokine FGF21 acts as an endocrine signal to oxytocin neurons, promoting neuronal activation and Oxt transcription and suppressing the simple sugar preference. SIRT1 promotes FGF21 signalling in oxytocin neurons and stimulates Oxt transcription through NRF2. Thus, neuronal SIRT1 contributes to the homeostatic regulation of macronutrient-based diet selection in mice.

\footnotetext{
${ }^{1}$ Laboratory of Metabolic Signal, Metabolic Signal Research Center, Institute for Molecular and Cellular Regulation, Gunma University, 3-39-15 Showa-machi, Maebashi, Gunma 371-8512, Japan. ${ }^{2}$ Advanced Scientific Research Leaders Development Unit, Gunma University, 3-39-15 Showa-machi, Maebashi, Gunma 371-8512, Japan. ${ }^{3}$ Frontier Research Core for Life Science, University of Toyama, 2630 Sugitani, Toyama, Toyama 930-0194, Japan. ${ }^{4}$ Department of Metabolism and Nutrition, Graduate School of Medicine and Pharmaceutical Science for Research, University of Toyama, 2630 Sugitani, Toyama, Toyama 930-0194, Japan. ${ }^{5}$ Department of Neuroscience II, Research Institute of Environmental Medicine, Nagoya University, Furocho, Nagoya 464-8601, Japan. ${ }^{6}$ Division of Brain and Neurophysiology, Department of Physiology, Jichi Medical University, 3311-1 Yakushiji, Shimotsuke, Tochigi 329-0498, Japan.

7 Department of Cell Biology, Graduate School of Medicine, Osaka University, 2-2 Yamadaoka, Suita, Osaka 565-0871, Japan. Correspondence and requests for materials should be addressed to T.S. (email: tsutomus@gunma-u.ac.jp) or to T.K. (email: kitamura@gunma-u.ac.jp)
} 
D iabetes and obesity are the third and fourth leading health risk factors, respectively, on a global scale ${ }^{1}$. Lifestyle modification, including diet therapy, comprises an important intervention against diabetes and obesity, but low adherence hinders its efficacy. The efficacy of diet therapy depends on both the quantity (caloric intake) and quality (macronutrient balance) of the ingested diet.

A large prospective epidemiological cohort study showed that excessive dietary carbohydrates impair health span and promote mortality and that replacing intake of carbohydrates with fat reduces mortality ${ }^{2}$. However, another study in overweight and obese men reported that replacing a high-carbohydrate baseline diet with an isocaloric low-carbohydrate ketogenic diet was not accompanied by increased body fat loss but it was associated with relatively small increases in energy expenditure ${ }^{3}$. Despite longstanding debates on the health benefits of fat restriction versus carbohydrate restriction ${ }^{4,5}$, both fat and carbohydrates (particularly simple sugars) are deemed highly rewarding. However, macronutrient selection behaviour cannot be fully understood by studying only hedonic feeding. For example, the central melanocortin system, which plays a major role in regulating caloric intake homeostatically, is implicated in the regulation of macronutrient preferences ${ }^{6-8}$, and some neuropeptides and endocrine signals regulate intake of specific macronutrients ${ }^{9}$. Nevertheless, an integrated understanding of the mechanisms that regulate macronutrient-based diet selection is lacking, yet it is required for developing a diet therapy that facilitates adherence.

To elucidate the homoeostatic regulation of macronutrient selection, we focused on the $\mathrm{NAD}^{+}$-dependent deacetylase, SIRT1. SIRT1 plays a dual role in controlling energy homoeostasis: SIRT1 in peripheral tissues promotes the use of fat as substrate, and SIRT1 in the central nervous system (CNS) promotes homoeostatic feeding control by improving hormone sensing ${ }^{10,11}$. Therefore, we hypothesized that CNS SIRT1 might regulate macronutrient intake by shifting the macronutrient preference to match the metabolic need in peripheral tissues (i.e. supply the appropriate substrate for use). SIRT1 is also important for prolonging lifespan in multiple organisms through a diet regimen called caloric restriction ${ }^{11}$. Across species, caloric restriction causes shifts in survival priorities from reproduction to self-maintenance, and simultaneously, this shift reprograms the metabolism from using carbohydrate to using fat ${ }^{12}$. This metabolic reprogramming is consistent with the priority shift to selfmaintenance, because glycolysis, which occurs at a high rate during carbohydrate use, is the metabolism of choice for replicating cells, including stem cells and cancer cells ${ }^{13,14}$. Therefore, our hypothesis is consistent with the evolutionarily-conserved principle that caloric restriction will shift metabolism to match the shift in survival priority.

By testing the hypothesis, here we show that neuronal SIRT1 shifts diet choice from sucrose to fat in mice, matching the peripheral metabolic shift. Mechanistically, neuronal SIRT1 regulates simple sugar preference through FGF21 and oxytocin signalling. Thus, neuronal SIRT1 contributes to the homoeostatic regulation of macronutrient-based diet selection in mice.

\section{Results}

Neuronal SIRT1 regulates macronutrient-based diet selection. To test whether central SIRT1 could simultaneously promote fat preference and suppress carbohydrate preference, we generated neuron-specific SIRT1 overexpression (NS-OE) and neuronspecific SIRT1 knockout (NS-KO) mice as neuron-specific SIRT1 gain-of-function and loss-of-function models, respectively. Briefly, neuron-specific Tau-Cre mice ${ }^{15}$ were crossed with either Rosa26-Sirt1 mice ${ }^{10}$ or Sirt1-flox mice ${ }^{16}$ to generate NS-OE and
NS-KO mice, respectively. We confirmed that hypothalamic Sirt1 expression levels in NS-OE and NS-KO mice were twofold and negligible, respectively, compared to those in wild-type littermates (Fig. 1a). The acetylation levels of SIRT1 substrates $\left(\right.$ FOXO1 ${ }^{17}, \mathrm{p} 53^{18,19}$ and $\mathrm{NF}-\mathrm{KB}^{20}$ ) in mice that received 3rd intracerebroventricular (ICV) injection of trichostatin A (TSA), an histone deacetylase inhibitor, were decreased in the hypothalamus of NS-OE mice (Supplementary Fig. 1a), confirming that NS-OE mice served as a SIRT1 gain-of-function model. Both modified mouse strains were viable, and food intake and body weight were not different from wild-type mice when fed normal chow (NC), a high-sucrose diet (HSD) or a high-fat diet (HFD), without a choice in diet (Fig. 1b-d; Supplementary Fig. 1b).

When NS-OE and NS-KO mice were given diet choices (NC vs. HSD or NC vs. HFD), they showed different preferences. The preference for the HSD was reduced in NS-OE and increased in NS-KO mice (Fig. 1e). Conversely, the preference for HFD was increased in NS-OE and reduced in NS-KO mice (Fig. 1f). In 5day experiments, these preferences resulted in significant differences in weight gain when mice selected between NC and HFD but not when they selected between NC and HSD (Supplementary Fig. 1c, d). Therefore, neuronal SIRT1 promoted fat preference and suppressed sucrose preference.

Neuronal SIRT1 regulates sucrose preference through oxytocin. If the general alteration in the hedonic system were the primary cause of the altered preferences for fat and sucrose in these modified mouse strains, then the preferences for both fat and sucrose would change in the same direction (instead of opposite directions), because they are both palatable to mice and drive reward circuits in the brain ${ }^{21}$. Therefore, we investigated the expression of genes known to encode neuropeptides and receptors that selectively regulate preference for a particular macronutrient ${ }^{9}$, such as neuropeptide $\mathrm{Y}(N p y)$, oxytocin $(O x t)$, galanin $(\mathrm{Gal})$ and neuromedin $\mathrm{U}(\mathrm{Nmu})$. We screened samples of the hypothalamus, ventral tegmental area (VTA), nucleus accumbens (NAc) and prefrontal cortex (PFC) from NS-KO and NS-OE mice. Hypothalamic expression of Oxt, which selectively suppresses the preference for carbohydrates but not fat ${ }^{22-27}$, was positively correlated with Sirt1 expression (Fig. 2a). SIRT1, however, did not affect the hypothalamic expression of Npy, NPY receptors Y1 and Y5 (Y1R and Y5R), Gal and Nmu (Supplementary Fig. 2a-e).

Next, we assessed the expression of Oxt receptor (Oxtr) in the hypothalamus, VTA, NAc and PFC of wild-type, NS-OE and NS$\mathrm{KO}$ mice. Oxtr expression levels were not significantly different between wild-type mice and NS-KO mice in any of these areas or between wild-type and NS-OE mice in any area except the NAc (Fig. 2b-e). To examine whether Oxt action on its receptor is necessary for the effect of neuronal SIRT1 on HSD preference, we blocked OXTRs using intraperitoneal (IP) injections of the OXTR antagonist L-368,899 (OXTR-A), which can penetrate the blood-brain barrier. OXTR-A treatment abolished the suppression of HSD intake in NS-OE mice (Fig. 2f). This result indicated that the action of Oxt on OXTR is required for neuronal SIRT1 to suppress the sucrose preference.

SIRT1 in Oxt neurons regulates preference for sucrose. Expression of Oxt positively correlated with that of Sirt1 in NS$\mathrm{OE}$ and NS-KO mice (Figs. 1a and 2a), which suggested that SIRT1 in Oxt neurons may regulate Oxt expression. Therefore, we next tested whether SIRT1 in Oxt neurons was sufficient to regulate sucrose preference. We genetically manipulated Sirt1 expression specifically in Oxt neurons by generating Oxt-neuronspecific SIRT1 overexpression (OS-OE) and Oxt-neuron-specific 
a

Hypothalamic Sirt1

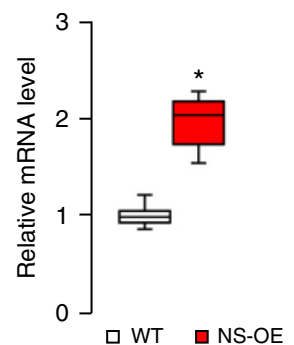

Hypothalamic Sirt1 (Exon4)

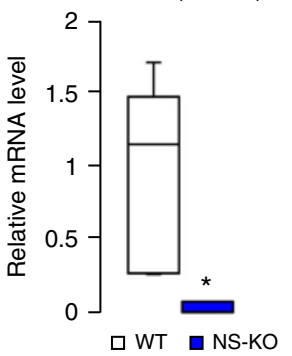

b

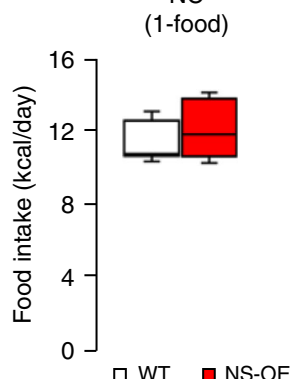

C

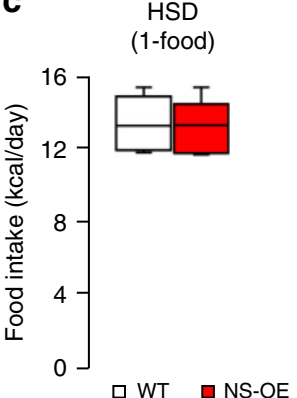

d $\quad H S D$

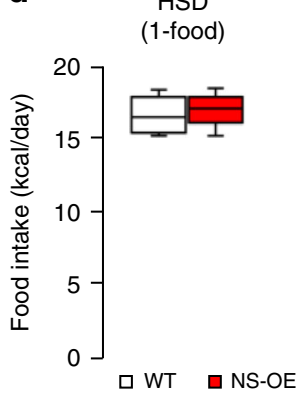

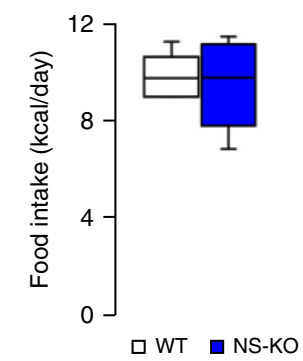
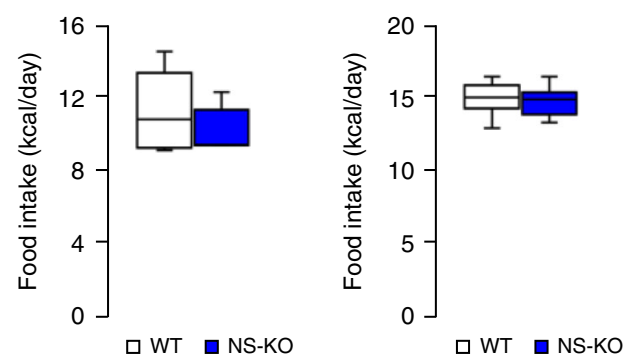

e
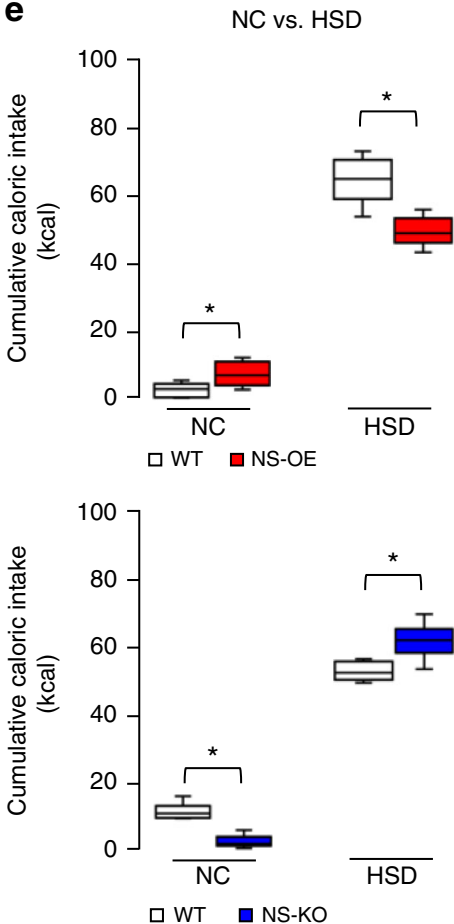

f
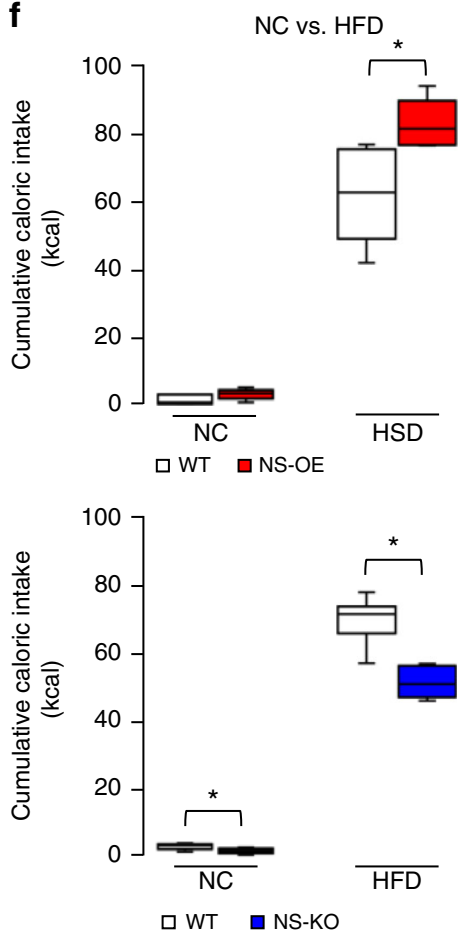

Fig. 1 Neuronal SIRT1 regulates macronutrient-based diet selection. a Hypothalamic Sirt1 mRNA expression of NS-OE (red) and NS-KO (blue) mice compared to wild-type mice (WT) ( $n=6$ per group). b-d Food intake (kcal/d) for NS-OE and NS-KO mice fed NC only (b), HSD only (c) and HFD only (d) ( $n=6$ per group). e, $\mathbf{f}$ Cumulative caloric intake for two-choice diets in 5 -day diet selection experiments: NC vs. HSD (e) and NC vs. HFD (f) ( $n=6$ per group). Data are shown as box and whisker plots (centre line, median; box limits, upper and lower quartiles; whiskers, the minimum and maximum value of a data set). ${ }^{*} p<0.05$. See also Supplementary Fig. 1

SIRT1 knockout (OS-KO) mice. Briefly, Oxt-neuron-specific Oxtires-Cre mice ${ }^{28}$ were crossed with either Rosa26-Sirt1 mice or Sirt1-flox mice to generate OS-OE and OS-KO mice, respectively. We performed immunohistochemistry to confirm that SIRT1 expression was manipulated specifically in Oxt neurons, and that the acetylation levels of SIRT1 substrates were decreased specifically in Oxt neurons of OS-OE mice that received 3rd ICV injection of TSA (Supplementary Fig. 3). We found that hypothalamic Oxt expression levels were increased and decreased in OS-OE and OS-KO mice, respectively, compared to those in wild-type littermates (Fig. 3a). Therefore, SIRT1 in Oxt neurons controlled Oxt expression.

In the absence of a dietary choice, the food intake and body weight of OS-OE and OS-KO mice were not significantly different from those of wild-type mice (Fig. 3b-d; Supplementary Fig. 4a). Moreover, the locomotor activity, metabolic rates and 
a
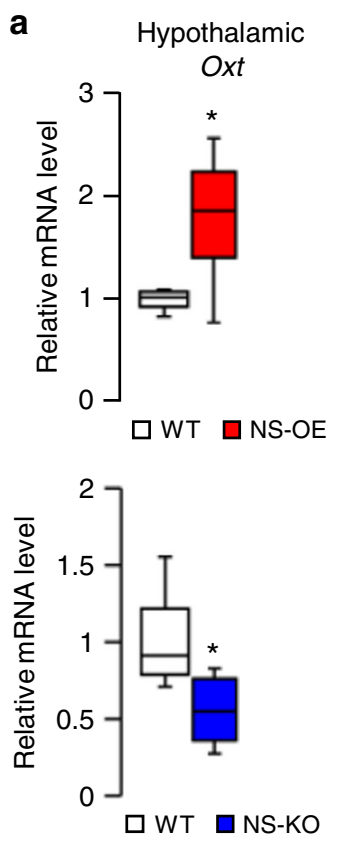

b
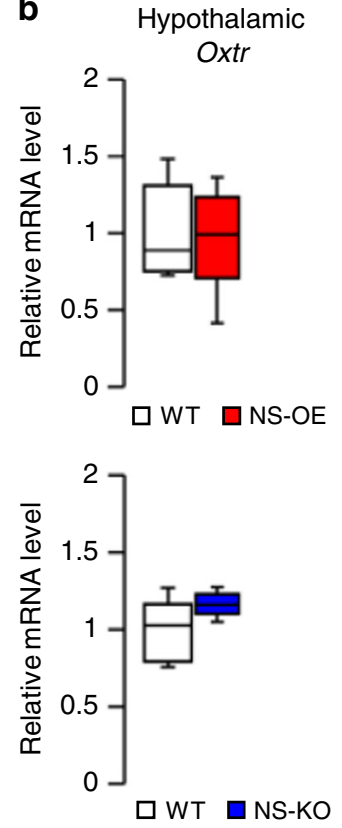

C

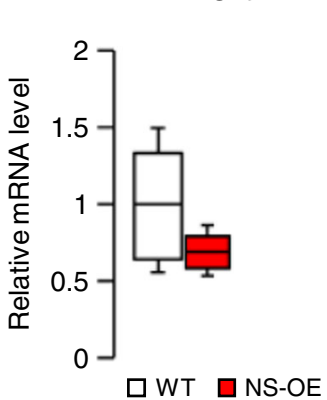

d
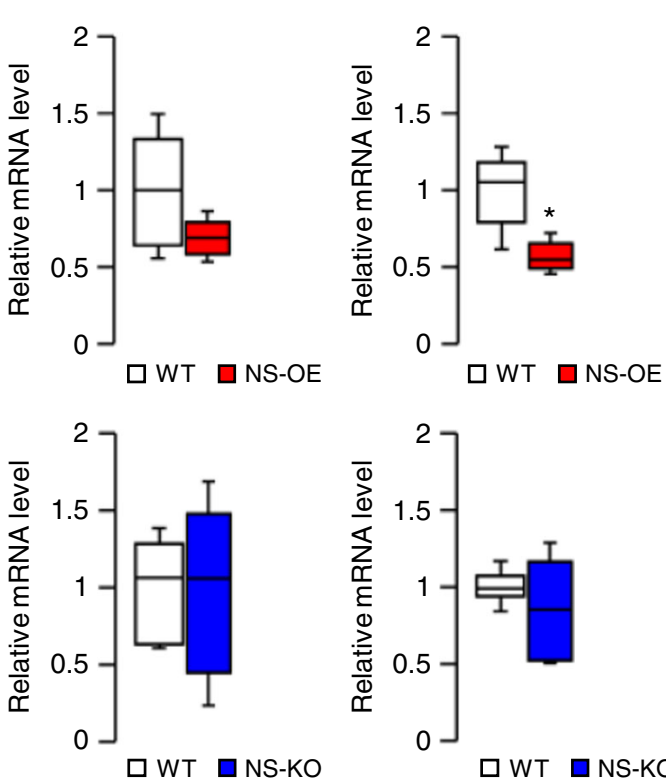

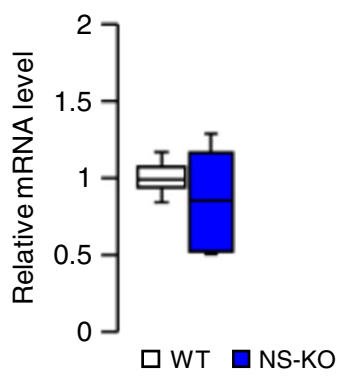

e
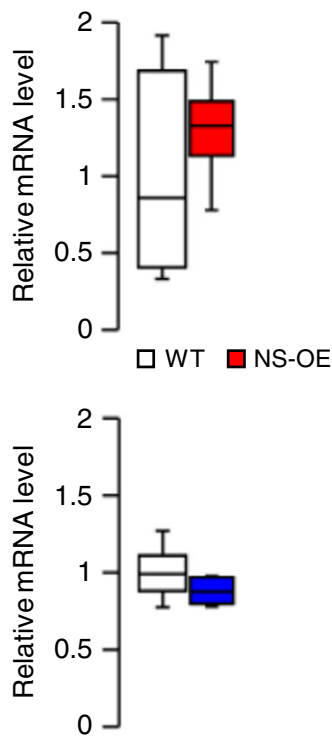

f

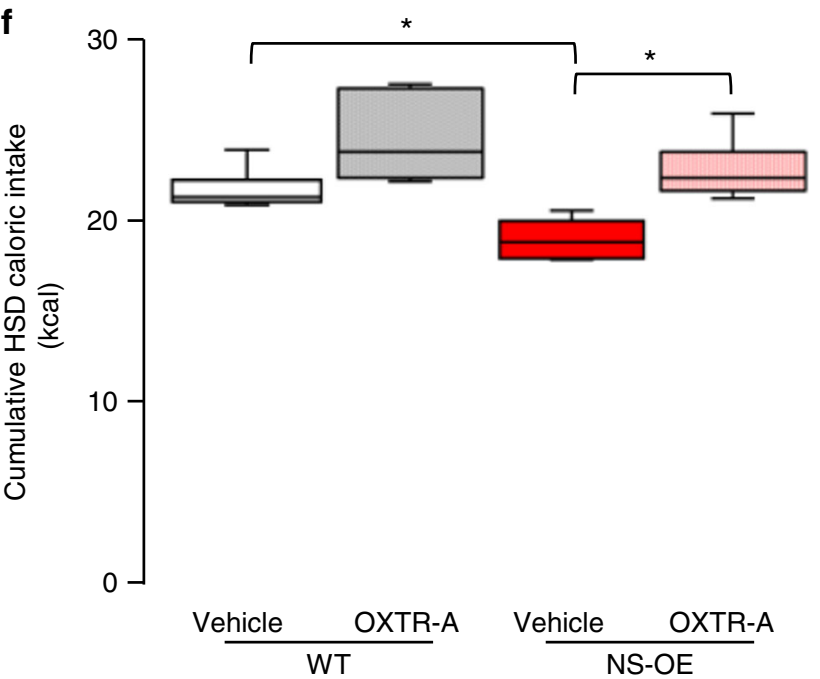

Fig. 2 Neuronal SIRT1 regulates sucrose preference through Oxt. a-e Relative RNA expression levels, for NS-OE (red) and NS-KO (blue) mice; Oxt expression in the hypothalamus (a); Oxtr expression in the hypothalamus (b), ventral tegmental (VTA) (c), nucleus accumbens (NAc) (d) and prefrontal cortex (PFC) (e) ( $n=6$ per group). $\mathbf{f}$ The effect of an IP injection of Oxt receptor antagonist OXTR-A (L-368,899) on HSD intake in NS-OE and littermate control mice ( $n=5$ per group). Data are shown as box and whisker plots (centre line, median; box limits, upper and lower quartiles; whiskers, the minimum and maximum value of a data set). ${ }^{\star} p<0.05$. See also Supplementary Fig. 2

plasma leptin levels of these mice were not significantly different from those of control mice (Supplementary Fig. $4 \mathrm{e}-\mathrm{j}$ ). On the other hand, when offered a choice between NC and HSD, the HSD preference was reduced in OS-OE mice and elevated in OS$\mathrm{KO}$ mice compared to wild-type mice, with similar results for males and females (Fig. 3e; Supplementary Fig. 4d). These findings were consistent with altered sucrose preferences observed in NS-OE and NS-KO mice under the diet-choice settings. Consequently, body weight was reduced and tended to be elevated in these groups, respectively, compared to wild-type mice over the 5-day experimental period (Supplementary Fig. 4b). Meanwhile, when offered a choice between NC and HFD, food intake and subsequent weight gain were not different between wild-type and either OS-OE or OS-KO mice (Fig. 3f; Supplementary Fig. 4c), which is consistent with the carbohydratespecific effect of Oxt on preference. Therefore, SIRT1 in Oxt neurons regulated preference for sucrose, but not fat, in these mice.

Next, we tested the necessity of the Oxt action on its receptor by OXTR antagonist. We found that OXTR-A treatment abolished the reduced HSD intake in OS-OE mice (Fig. 3g). We then tested the necessity of the neural activation of Oxt neurons for the reduced HSD intake in OS-OE mice by using an inhibitory designer receptor exclusively activated by designer drugs (DREADDs) ${ }^{29}$ in Oxt neurons in the paraventricular nucleus of the hypothalamus $(\mathrm{PVH})$. The inhibitory DREADD receptor hM4Di, when stimulated by the DREADD ligand clozapine, activates G-protein inwardly rectifying potassium channels, thereby hyperpolarizing and attenuating neuronal activity ${ }^{29}$. We infected AAV2-hSyn-FLEX-mCherry (control) or AAV2-hSyn-FLEX-hM4Di-mCherry (hM4Di) in PVH Oxt neurons of OS-OE mice. These mice have the Oxt-ires-Cre allele, 


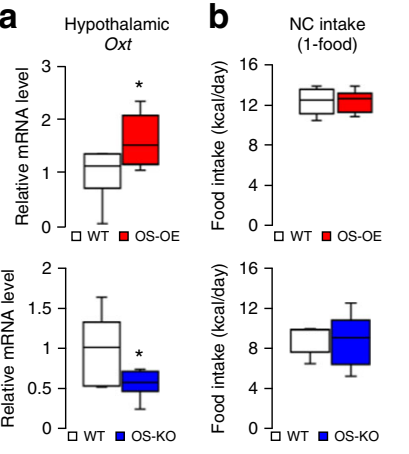

e
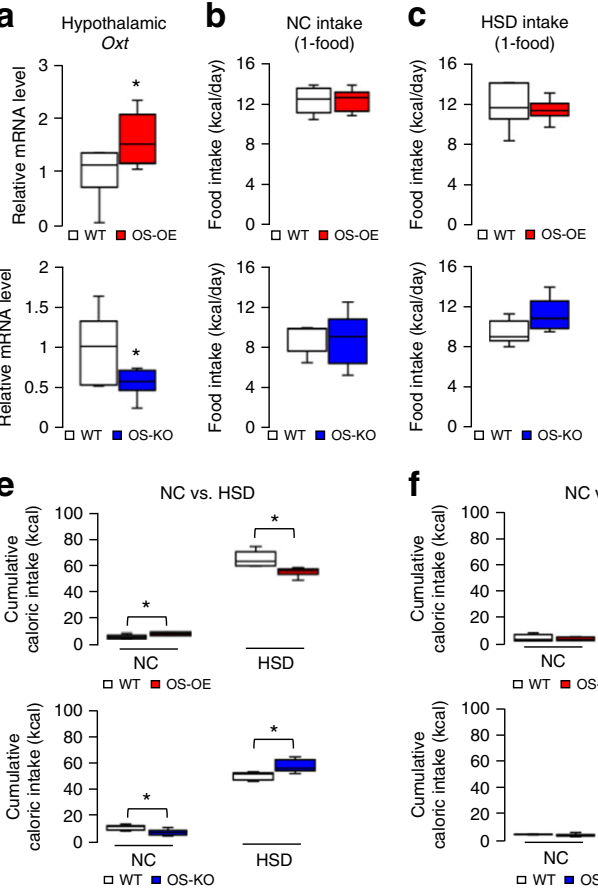

d HFD intake
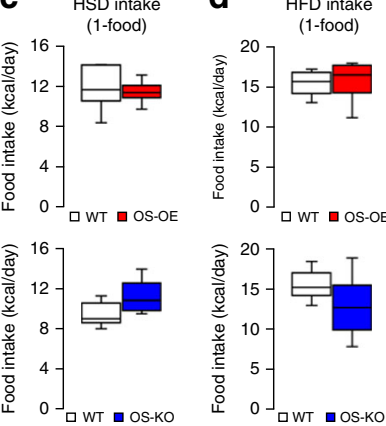

f
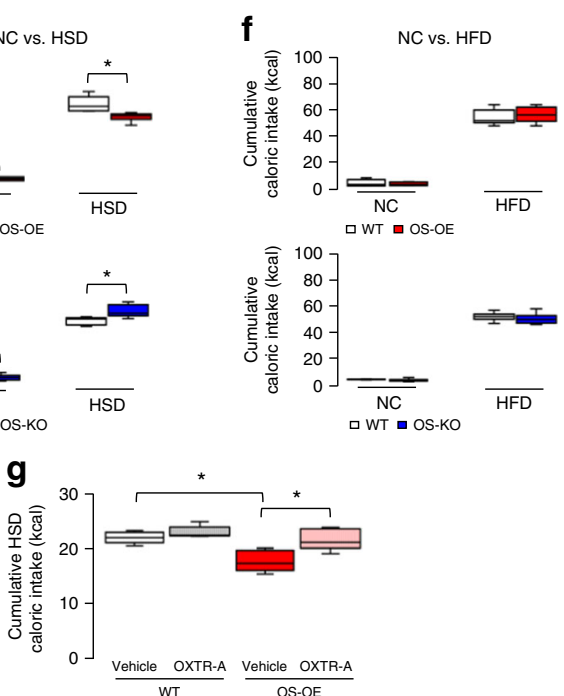

h

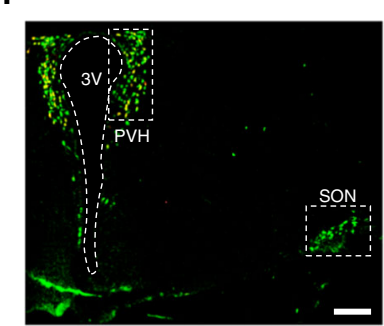

i
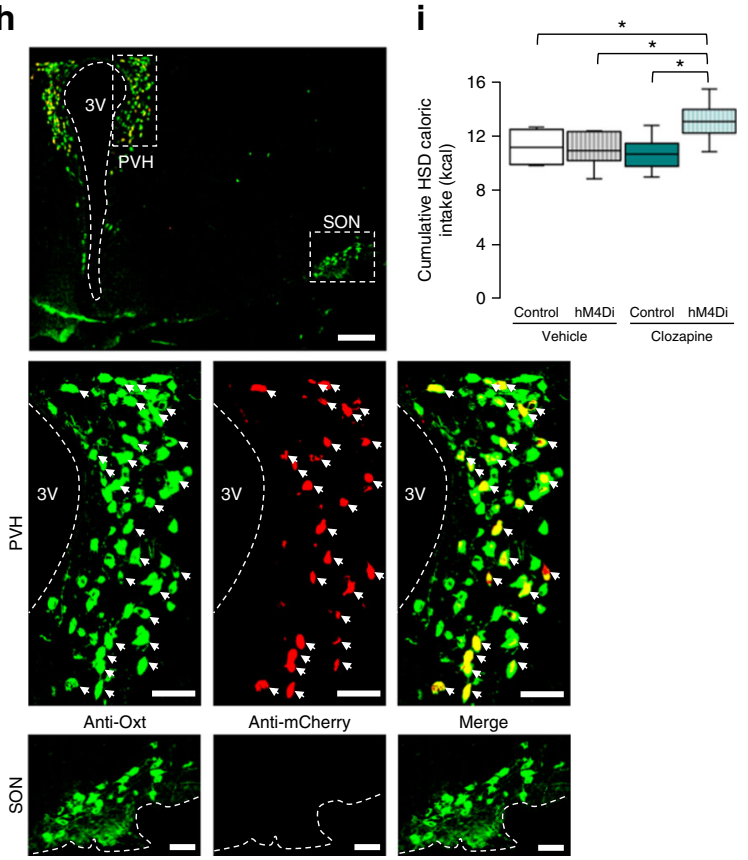

and recombination of the FLEX vectors by the Cre recombinase would be expected to induce expression of the target genes (mCherry or hM4Di-mCherry) only in Cre-positive PVH Oxt neurons. We confirmed that the control or hM4Di was selectively expressed in Oxt neurons located in the PVH, but not in the supraoptic nucleus (SON), based on the co-localization of signals emitted from anti-mCherry stains and anti-Oxt stains (Fig. 3h).

Fig. 3 SIRT1 in Oxt neurons regulates sucrose preference. a Hypothalamic Oxt mRNA expression in WT, OS-OE (red) and OS-KO (blue) mice $(n=6$ per group). b-d Food intake (kcal/d) for OS-OE and OS-KO mice fed NC only (b), HSD only (c) and HFD only (d) ( $n=6$ per group). e, $\mathbf{f}$ Cumulative caloric intake for two-choice diets in 5-day diet selection experiments: NC vs. HSD (e) and NC vs. HFD (f) ( $n=6$ per group). $\mathbf{g}$ The effect of an IP injection of the Oxt receptor antagonist OXTR-A (L-368,899) on HSD intake in OS-OE and littermate control (WT) mice ( $n=6$ per group). h Representative immunofluorescent images of the paraventricular nucleus (PVH) and the supraoptic nucleus (SON) of the hypothalamus of OS-OE mice used in the DREADD experiments. Co-localization of Oxt (green) and mCherry (red) signals is seen in the merged image (white arrows). The size of the scale bar is $200 \mu \mathrm{m}$ for the low magnification panel and $50 \mu \mathrm{m}$ for the high magnification panels. i Clozapine-induced inhibition of Oxt neuronal activity by hM4Di increased HSD intake in OS-OE mice. The changes in HSD intake (clozapine-vehicle) during the course of 3-day NC vs. HSD feeding ( $n=8$ per group). Data are shown as box and whisker plots (centre line, median; box limits, upper and lower quartiles; whiskers, the minimum and maximum value of a data set). ${ }^{*} p<0.05 .3 \mathrm{~V}, 3 \mathrm{rd}$ ventricle. See also Supplementary Figs. 3 and 4

We injected IP clozapine $(1 \mu \mathrm{g} / \mathrm{kg}$ ) or vehicle (phosphate-buffered saline (PBS); $\mathrm{pH} 7.4$ ) in OS-OE mice expressing control or hM4Di. Injection of clozapine into hM4Di-expressing OS-OE mice increased HSD intake compared to that of vehicle injection (Fig. 3i). On the other hand, clozapine injection to controlexpressing OS-OE mice did not significantly alter HSD intake as compared to that of vehicle injection (Fig. 3i). Therefore, activation of Oxt neurons mediates the effect of SIRT1 in suppressing sucrose preference in OS-OE mice. These results collectively indicated that SIRT1 in Oxt neurons is sufficient to suppress the sucrose preference and that the effect of SIRT1 on sucrose preference is mediated by released Oxt upon neural activation and its action on OXTR.

SIRT1 in Oxt neurons regulates simple sugar preference. We next asked if the preference alteration in OS-OE and OS-KO mice was specific to sucrose or if the phenomenon could be generalized to all carbohydrates. We addressed these questions by analysing the preference phenotypes of OS-OE and OS-KO mice relative to various carbohydrates using two-bottle choice tests over 3 days. These experiments revealed that the preference phenotype was specific for simple sugars (glucose, fructose and sucrose) (Fig. 4a-c), and not for complex sugars (dextrin) (Fig. 4d).

Preference for simple sugars could be mediated by orosensory stimuli such as taste and by a post-ingestive metabolic effect of sugars. To address the effect of taste versus the post-ingestive metabolic effect of sugars, we performed brief two-bottle choice tests over $10 \mathrm{~min}$, which minimized the post-ingestive metabolic effect. However, when we conducted these brief tests with sucrose, both OS-OE and OS-KO mice showed no significant differences from wild-type mice (Fig. 4e). We next tested the artificial sweeteners, saccharin and aspartame, in sugar-naive mice. We used sugar-naive mice to avoid the potential post-ingestive metabolic effect of non-nutritive artificial sweetener induced by conditioning-based learning ${ }^{30,31}$. Twobottle choice tests with these sweeteners revealed that preferences to the sweeteners were not different in OS-OE mice and OS-KO mice in the 3-day tests (Fig. 4f, g). There was no significant difference in the preference for bitter-tasting quinine in these mice (Fig. 4h). These data collectively indicated that SIRT1 regulated the preference only for simple sugars, and that the taste effect of sugar did not contribute to the SIRT1 regulation of the simple sugar preference. Therefore, the metabolic effects of sugars 
a
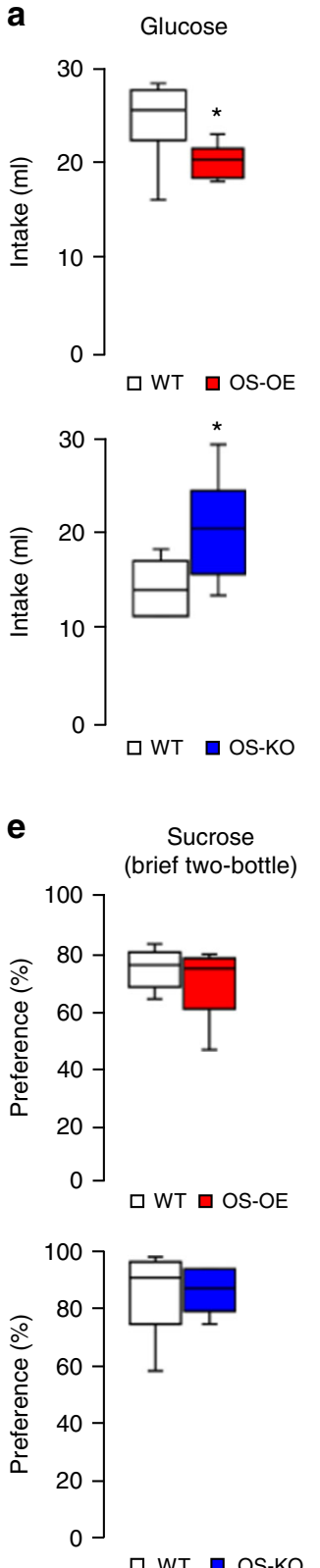

b
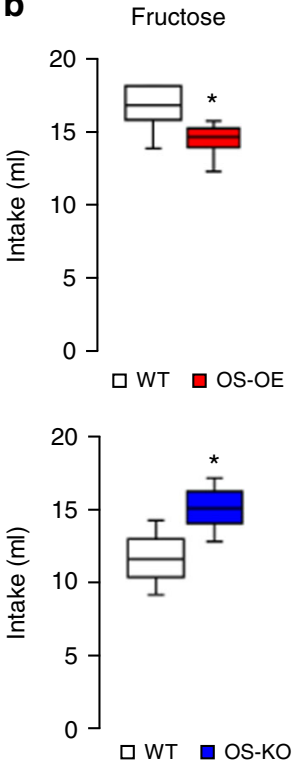

f
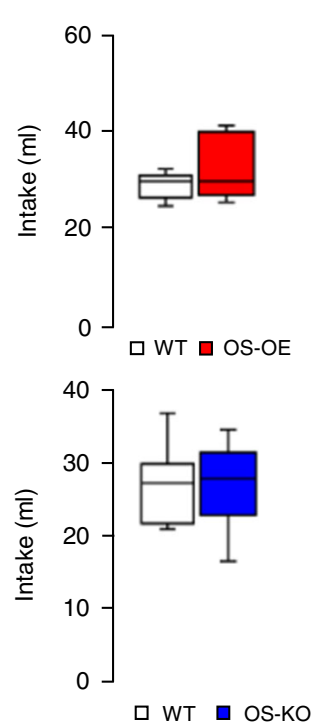

c
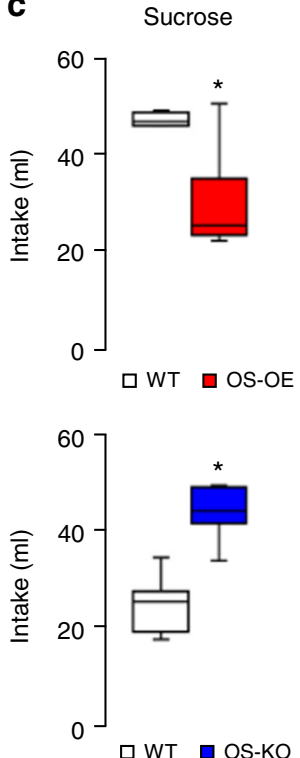

g Aspartame
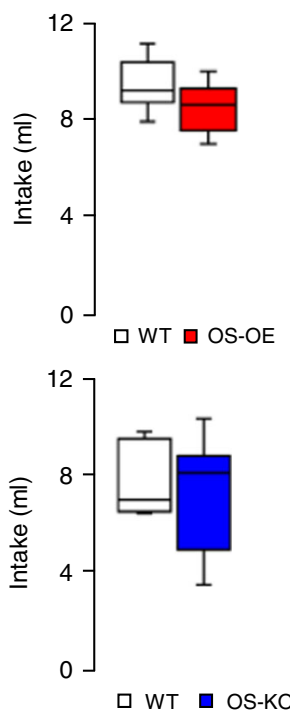

d Dextrin
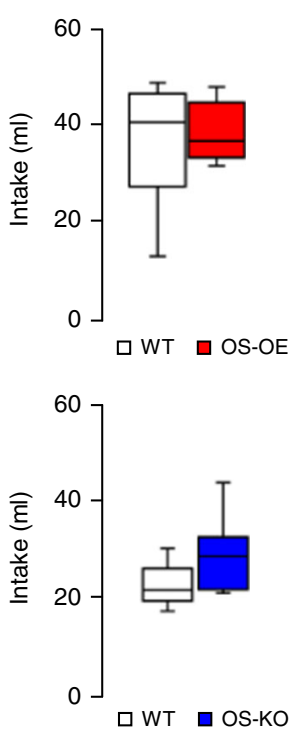

h
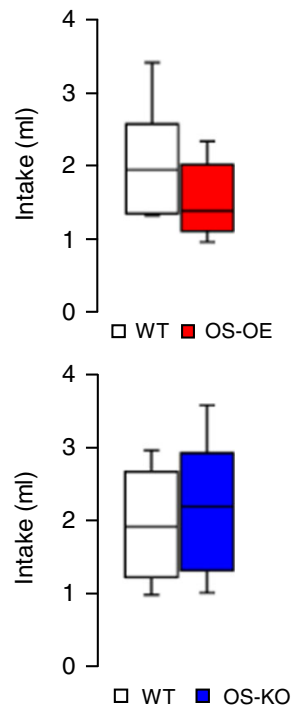

Fig. 4 SIRT1 in Oxt neurons specifically regulates preferences for simple sugars. Two-bottle choice tests in OS-OE (red) and OS-KO (blue) mice. a-d Results of 3-day two-bottle choice tests ( $100 \mathrm{mM}$ glucose (a), $100 \mathrm{mM}$ fructose (b), $100 \mathrm{mM}$ sucrose (c) and $2 \%$ dextrin (d)) ( $n=7 \mathrm{in}$ the WT group; $n=6$ in the OS-OE and OS-KO groups). e Results of 10-min brief access two-bottle choice tests for $100 \mathrm{mM}$ sucrose over water ( $n=6$ per group). $\mathbf{f}, \mathbf{g}$ Results of two-bottle choice tests with artificial sweeteners ( $0.2 \%$ saccharin $(\mathbf{f})$ and $0.2 \%$ aspartame $(\mathbf{g})$ ) over water, given for 3 days in sugar-naive mice $(n=8$ per group). h Results of 3-day two-bottle choice tests with $1.5 \mathrm{mM}$ quinine (bitter taste) ( $n=7$ in the WT group; $n=6$ in the OS-OE and OSKO groups). Data are shown as box and whisker plots (centre line, median; box limits, upper and lower quartiles; whiskers, the minimum and maximum value of a data set). ${ }^{\star} p<0.05$. See also Supplementary Fig. 9

played predominant roles in the SIRT1 regulation of the simple sugar preference.

Oxt neurons in the PVH respond to FGF21. If SIRT1 regulates sucrose preference through Oxt and the effect is mediated primarily by the post-ingestive metabolic effect of sugars, metabolic signals that represent simple sugar ingestion may activate Oxt neurons. FGF21 is secreted from the liver upon simple sugar ingestion in rodents and humans ${ }^{32,33}$, and it suppresses simple sugar intake and preference by signalling to the $\mathrm{PVH}^{32,34}$. The PVH harbours numerous subtypes of peptidergic neurons, including Oxt neurons ${ }^{35}$. The expression of the neural activation marker, c-Fos, in PVH Oxt neurons was induced by oral ingestion of sucrose $\mathrm{e}^{27}$ and intra-gastric ingestion of sweetened condensed milk ${ }^{36}$. Therefore, we hypothesized that FGF21 secreted upon simple sugar ingestion may be the metabolic signal to Oxt neurons, which control simple sugar preference by way of negative feedback.

For FGF21 to work as a metabolic feedback signal to Oxt neurons, these cells must express FGF21 receptors. FGF21 receptor is composed of $\beta$-Klotho $(\mathrm{Klb})$ and the FGF receptor heterodimer. The distribution of FGF21 receptors is defined by the restricted expression pattern of $\beta$-Klotho because FGF 


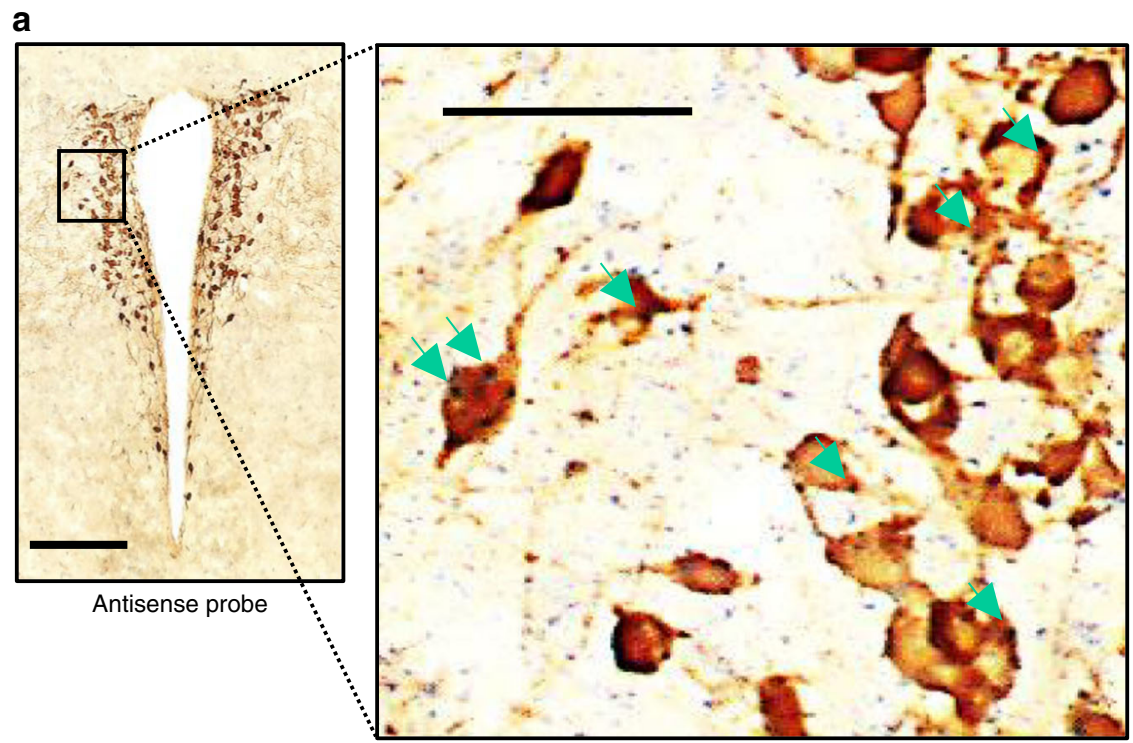

b

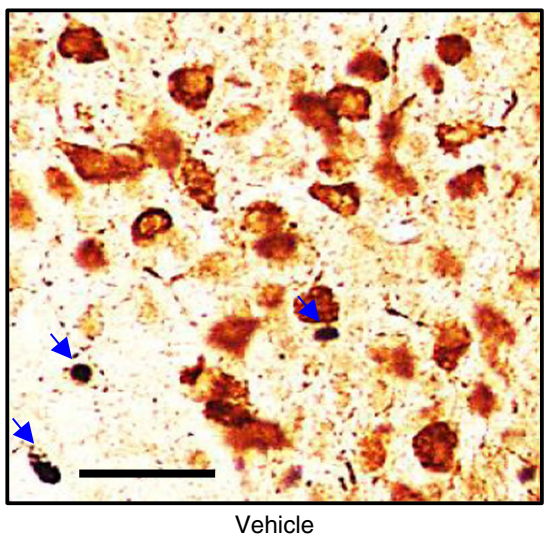

d

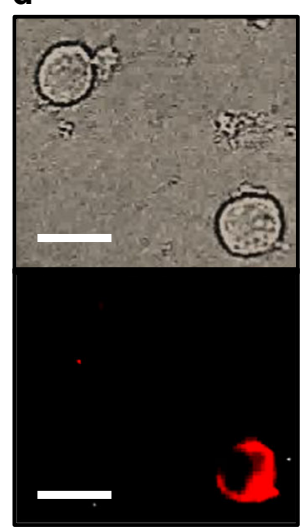

e

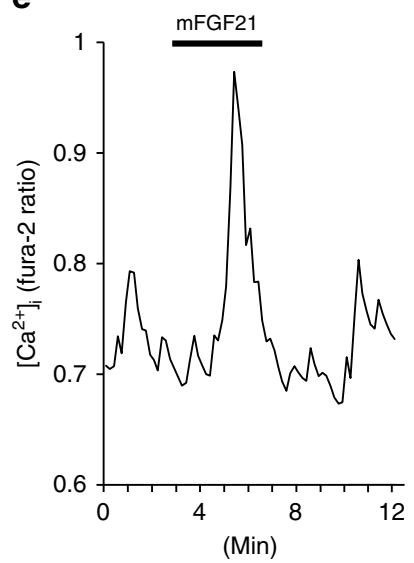

f

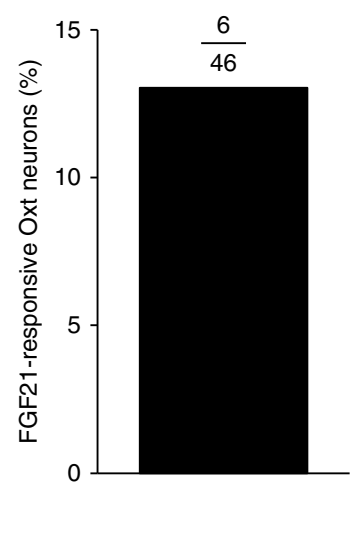

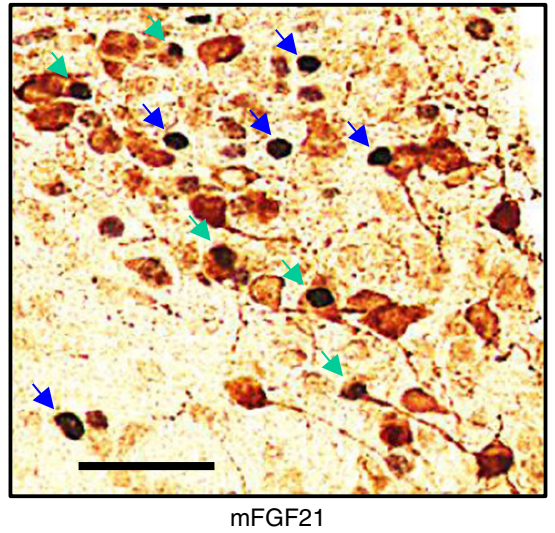

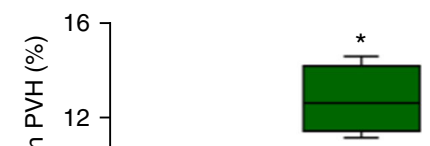

g

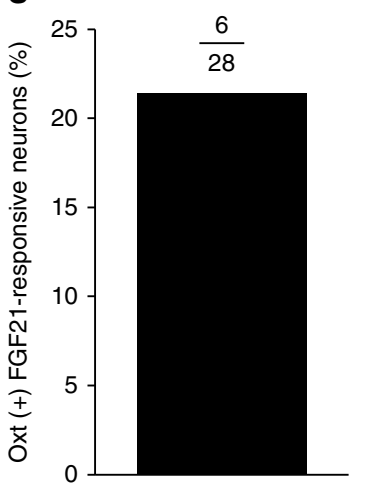

receptors are expressed ubiquitously $y^{37}$. Therefore, we checked for the co-localization of $K l b$ and $O x t$ expression by performing in situ hybridization and immunohistochemistry on PVH sections. We detected Klb mRNA staining in some Oxtimmunoreactive neurons (Fig. 5a; Supplementary Fig. 5a). Oxt neurons are also present in the $\mathrm{SON}$, and we also detected $\mathrm{Klb}$ mRNA staining in some Oxt-immunoreactive SON neurons (Supplementary Fig. 5b). We next tested whether FGF21 could activate Oxt neurons in vivo. An IP injection of murine FGF21
(mFGF21) induced c-Fos expression in 13\% of the PVH Oxt neurons (Fig. 5b, c), including both magnocellular and parvocellular Oxt neurons. On the other hand, mFGF21 did not significantly induce c-Fos expression in SON Oxt neurons (Supplementary Fig. $5 c-d$ ). Therefore, a fraction of Oxt neurons represented one of the targets of FGF21 in the PVH.

Neuronal activation by a hormone in vivo, however, can be triggered through the direct action of the hormone on the target neuron and also by indirect action through neural circuits. 
Fig. 5 Oxt neurons in the PVH respond to FGF21. a Photomicrographs depict coronal PVH sections from wild-type C57BL/6 mice. Sections were subjected to in situ hybridization to identify $\beta$-Klotho mRNA ( $K I b$, blue dots). Then, sections were immunostained to identify Oxt protein (brown) expression. Green arrows indicate KIb mRNA staining in Oxt neurons. b Photomicrographs depict coronal PVH sections from wild-type C57BL/6 mice injected with murine FGF21 (mFGF21) (1 mg/kg, IP) or water (vehicle). The sections were immunostained for c-Fos (black) and Oxt (brown). Blue arrows indicate Oxt ( - ) c-Fos $(+)$ neurons; green arrows indicate c-Fos $(+)$ Oxt $(+)$ neurons. c The percentage of c-Fos $(+)$ cells among PVH Oxt neurons after an IP injection of vehicle or mFGF21 ( $n=4$ per group). d, e A representative $\left[\mathrm{Ca}^{2+}\right]_{i}$ result, expressed by fura-2 fluorescence ratio (F340/F380), in an ex vivo single-cell Oxtneuron. Addition of $50 \mathrm{ng} / \mathrm{ml} \mathrm{mFGF} 21$ for $5 \mathrm{~min}$ to perfusate (KRB containing $10 \mathrm{mM}$ glucose) increased $\left[\mathrm{Ca}^{2+}\right]_{i}$ in a single PVH neuron (e), which was subsequently shown to be IF to Oxt by immunocytochemical staining (d). $\mathbf{f}, \mathbf{g}$ Percentage of mFGF21-responding Oxt neurons among PVH Oxt neurons (f) and mFGF21-responding PVH neurons (g). The number above the bar indicates the number of neurons that responded over number examined. The length of scale bar is $200 \mu \mathrm{m}$ for the low magnification panel and $50 \mu \mathrm{m}$ for the high magnification panel in $\mathbf{a} ; 50 \mu \mathrm{m}$ in $\mathbf{b}$; and $20 \mu \mathrm{m}$ in d. Data are shown as box and whisker plots (centre line, median; box limits, upper and lower quartiles; whiskers, the minimum and maximum value of a data set). ${ }^{\star} p<0.05$. See also Supplementary Fig. 5

Therefore, we examined whether FGF21 directly activates PVH Oxt neurons using ex vivo calcium imaging of the dispersed single-cell PVH neurons, so that the contribution of neural circuits to neural activation was eliminated. Treatment of $\mathrm{mFGF} 21(50 \mathrm{ng} / \mathrm{ml})$ increased intracellular calcium concentration $\left(\left[\mathrm{Ca}^{2+}\right]_{\mathrm{i}}\right)$ in the PVH neurons that were subsequently shown to be immunofluorescent (IF) to Oxt (Fig. 5d, e). Of $46 \mathrm{PVH} \mathrm{Oxt}$ neurons, 6 of them $(13 \%)$ showed increased $\left[\mathrm{Ca}^{2+}\right]_{\mathrm{i}}$ in response to mFGF21 (Fig. 5f), while 6 out of 28 (21\%) mFGF21-activated $\mathrm{PVH}$ neurons were Oxt-positive (Fig. 5g). These results showed that FGF21 can directly activate some PVH Oxt neurons ex vivo.

This evidence collectively suggested that FGF21 stimulates Oxt neuronal activity linked to Oxt release at axon terminals. Although Oxt is also released from Oxt neuronal somata and dendrites independent of an action potential ${ }^{38}$, FGF21 failed to induce significant somato-dendritic release of Oxt (Supplementary Fig. 5e). Therefore, we speculate that FGF21 stimulates Oxt neurons and that release of Oxt at projection site(s) mediates the feedback suppression of simple sugar preference by FGF21.

FGF21 regulates Oxt expression via AKT. Because sucrose ingestion also induces Oxt expression as well as Oxt neural activation $^{27}$, we next analysed the effects of FGF21 on Oxt expression in vivo and in vitro. An IP injection of mFGF21 induced Oxt expression in the mouse hypothalamus (Fig. 6a). When cells from the mouse hypothalamic N41 cell line were treated with mFGF21, Oxt expression and Oxt promoter $(-5000$ bp) activity were also induced (Fig. 6b-d). Binding of FGF21 to its receptor (KLB/FGFR heterodimer) activates ERK and AKT signalling ${ }^{37}$, and these signalling pathways were also activated by mFGF21 in N41 cells (Fig. 6e). FGF21-induced Oxt expression was blocked by adding wortmannin (a PI3K inhibitor, which blocks subsequent AKT activation), but not by UO126 (an ERK1/ 2 inhibitor) (Fig. 6f). Therefore, FGF21-dependent Oxt expression is mediated by FGF21-KLB-AKT signalling. Because ERK phosphorylation is used as a marker of neural activation, FGF21 stimulated both neural activation and Oxt expression in Oxt neurons through these two kinases that act downstream of the FGF21 receptor.

SIRT1 promotes Oxt expression via FGF21-KLB-AKT signalling. Our results indicated that SIRT1 regulates simple sugar preference through Oxt and that FGF21 signalling regulates Oxt. Therefore, we hypothesized that SIRT1 may regulate FGF21-Oxt signalling. To analyse the mechanism linking SIRT1 to this FGF21-Oxt signalling, we manipulated SIRT1 levels using viral vectors in the hypothalamic N41 cell line. The level of Sirt1 expression was positively correlated with levels of Oxt and Klb expression, but FGF receptor $1(F g f r 1)$ and Oxtr expression was not affected (Fig. 7a-d; Supplementary Fig. 6a). In addition, the expression of Sirt1 positively correlated with that of $K l b$ but not of Fgfrl in NS-OE and NS-KO mice hypothalami (Fig. 7e, f), suggesting that SIRT1 promotes $\mathrm{Klb}$ expression both in vitro and in vivo. Moreover, SIRT1 and FGF21 cooperatively regulated Oxt expression in N41 cells (Fig. 7g, h). Additionally, hypothalamic Oxt expression was further increased by IP injection of mFGF21 into OS-OE mice but not OS-KO mice (Fig. 7i), suggesting that SIRT1 in Oxt neurons facilitates FGF21-induced Oxt expression in vivo. Moreover, SIRT1 overexpression enhanced FGF21induced AKT phosphorylation, and a Sirt1 knockdown suppressed it in N41 cells (Fig. 7j, k). Therefore, SIRT1 facilitates FGF21 signalling in Oxt neurons and promotes Oxt expression.

Serum FGF21 levels in OS-OE and OS-KO mice were not significantly different from the levels in control mice, either in the ad libitum fed state or $1 \mathrm{~h}$ after sucrose ingestion (Supplementary Fig. $6 \mathrm{~b}, \mathrm{c}$ ). Therefore, we concluded that simple sugar preference phenotypes in these mice were driven mainly by the altered sensitivity of Oxt neurons to FGF21 and not by changes in the circulating FGF21 levels.

SIRT1 regulates the expression of Oxt through NRF2. To elucidate the mechanisms of transcriptional regulation of the Oxt gene, we performed promoter activity assays. Specifically, we aimed to identify the transcription factor(s) responsible for the regulation of Oxt expression by SIRT1 and FGF21-KLB-AKT signalling. SIRT1 overexpression enhanced the activity of the -5000 bp Oxt promoter (Fig. 8a). DNA deletion studies revealed that a region between -2340 and -2000 bp upstream of the Oxt gene is responsible for SIRT1-dependent promoter activation (Fig. 8b, c). Motif analyses showed that this region contains the antioxidant-response element (ARE), which binds the transcription factor NF-E2-related factor $2(\mathrm{NRF} 2)^{39}$. A previous study showed that AKT activation by FGF21 promotes the nuclear translocation and subsequent activation of NRF2 ${ }^{40}$. We confirmed that the nuclear NRF2 level was increased with SIRT1 overexpression and decreased with a Sirt1 knockdown in N41 cells (Fig. $7 \mathrm{~d}$ ).

To address the role of NRF2 in the transcriptional regulation of Oxt by SIRT1 and FGF21, we performed a series of experiments. Oxt mRNA expression was significantly upregulated with the NRF2 activator, diethyl maleate (DEM) (Supplementary Fig. 7a, b). A knockdown of NRF2 expression prevented SIRT1-induced Oxt expression (Fig. 7e-g). Both SIRT1 overexpression and FGF21 treatment promoted NRF2 recruitment to the single ARE on the Oxt promoter (Fig. 8h; Supplementary Fig. 7c), which was detected by chromatin immunoprecipitation (ChIP) assays. Finally, point mutations in the single ARE motif within the -2340 bp Oxt promoter construct were sufficient to block SIRT1, FGF21 or DEMdependent promoter activation (Fig. 8i; Supplementary Fig. 7d, e). Therefore, these data collectively show that SIRT1 promotes Oxt expression through FGF21-KLB-AKT-NRF2 signalling. 
a

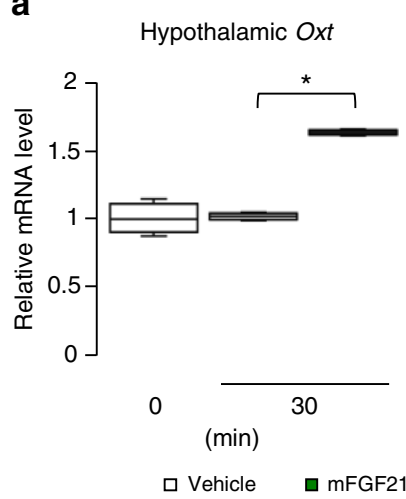

b

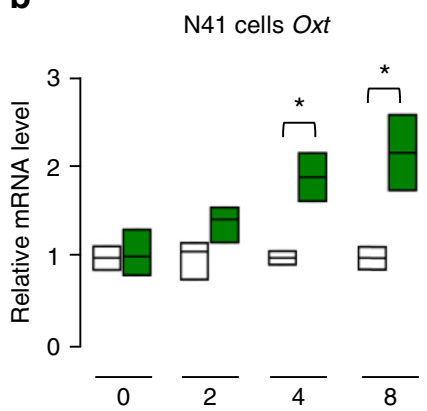

(h)
C

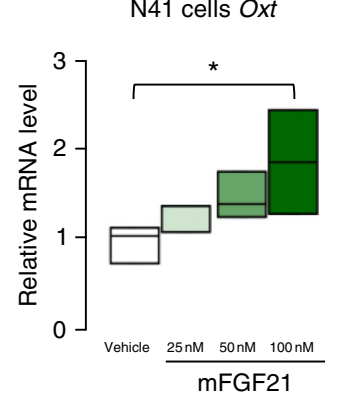

d N41 cells Oxt promoter (-5000 bp)

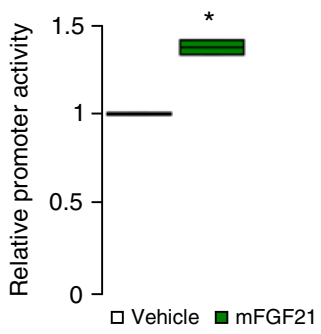

$\square$ Vehicle $\square$ mFGF21

e

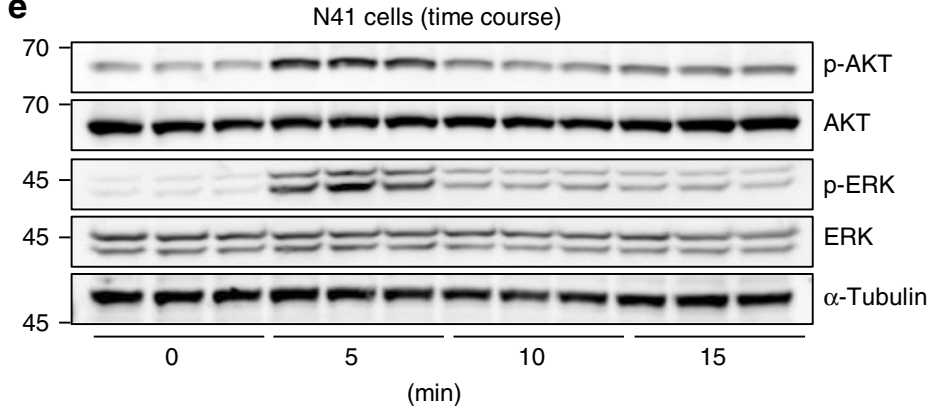

f

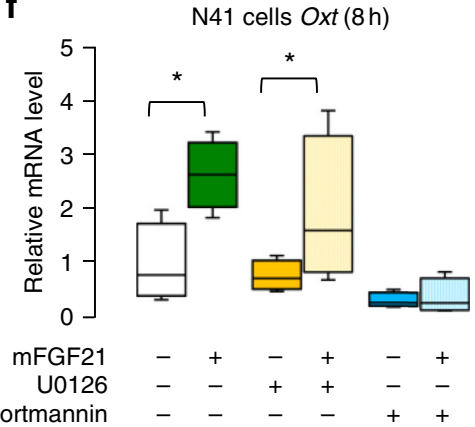

Fig. 6 FGF21 stimulates Oxt expression through AKT. a Hypothalamic Oxt mRNA expression levels after IP injection of mFGF21 (1 mg/kg) or water (vehicle) ( $n=4$ per group). b, c The relative expression of Oxt mRNA in hypothalamic N41 cells after mFGF21 treatment: time-course data (b) and doseresponse data (c) ( $n=3$ per group). d Luciferase activity of Oxt promoter of -5000 bp in $N 41$ cells was measured in response to $100 \mathrm{nM}$ mFGF21 and

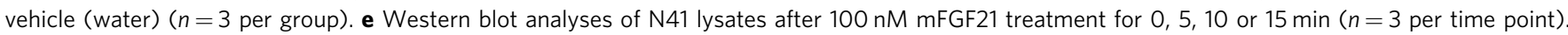
f Effect of inhibiting ERK signalling or AKT signalling on FGF21-induced Oxt expression. N41 cells underwent 30-min pre-treatment with or without the ERK inhibitor (10 $\mu \mathrm{M}$ U0126) or the PI3K inhibitor (10 $\mu \mathrm{M}$ wortmannin); subsequently, cells were incubated for $8 \mathrm{~h}$ in the absence or presence of $100 \mathrm{nM}$ mFGF21 ( $n=4$ per group). Data are shown as box and whisker plots (centre line, median; box limits, upper and lower quartiles; whiskers, the minimum and maximum value of a data set). ${ }^{*} p<0.05$. Data in $\mathbf{a}, \mathbf{b}, \mathbf{c}, \mathbf{d}, \mathbf{f}$ were replicated more than three times, and data in e were replicated twice in the laboratory

Fasting attenuates FGF21-induced Oxt expression through KLB. We found the link between FGF21 and Oxt by studying simple sugar preference phenotypes regulated by SIRT1. However, in addition to simple sugar ingestion, plasma FGF21 levels are elevated by fasting ${ }^{37}$, so we analysed the effect of fasting on expression levels of Klb and Oxt and hypothalamic levels of NAD ${ }^{+}$. Although it is generally assumed that SIRT1 is active during fasting, it has been shown that fasting had differential effects on SIRT1 expression among different tissues ${ }^{41}$. Even within the brain, fasting increased SIRT1 protein levels in the cerebral cortex, but it reduced SIRT1 levels in the hypothalamus ${ }^{42}$. Moreover, we found that fasting also significantly reduced hypothalamic $\mathrm{NAD}^{+}$(Fig. 9a); consequently, as expected, fasting (not caloric restriction) reduced the estimated total hypothalamic SIRT1 activity (estimated by the abundances of SIRT1 protein and its cofactor $\mathrm{NAD}^{+}$), leading to increased acetylation levels of SIRT1 substrates (Fig. 9b). It also was associated with reduced expression of $\mathrm{Klb}$ and $\mathrm{Oxt}$ (Fig. 9c, d). In contrast to the FGF21induced Oxt expression in fed mice (Fig. 6a), the FGF21-induced Oxt expression was not observed in fasted mice (Fig. 9e), indicating that the sensitivity of the hypothalamus to FGF21 is reduced during fasting in association with the reduced expression of FGF21 receptors (due to reduced KLB) (Fig. 9f).

On the other hand, HSD and HFD feeding showed no effect on hypothalamic Sirt1 mRNA, SIRT1 protein, NAD ${ }^{+}$levels or the acetylation levels of SIRT1 substrates compared to NC feeding in wild-type C57BL/6 male mice (Supplementary Fig. 8a-d). Consequently, under the fed condition (when hypothalamic SIRT1 activity was intact), the FGF21-Oxt signalling pathway was intact, and it negatively regulated the simple sugar preference through Oxt (Fig. 9e). SIRT1 may serve as a tuner for FGF21 sensitivity in Oxt neurons and regulate simple sugar preference in a contextdependent manner.

\section{Discussion}

We identified SIRT1 as a regulator of macronutrient preference and found that it regulates simple sugar preference through FGF21-NRF2-Oxt signalling (Fig. 10). Oxt neurons were identified as one of the in vivo targets of FGF21, and FGF21 promoted Oxt neuronal activation via ERK signalling and Oxt expression via AKT signalling. SIRT1 promoted FGF21 sensitivity in Oxt neurons and enhanced negative feedback regulation of simple sugar preference. Thus, our findings extend understanding of the homoeostatic regulation of macronutrient preference. Considering the importance of macronutrient balance on health span and weight control, greater understanding of this aspect of feeding regulation would help with elucidating the physiology and pathophysiology of feeding behaviour in relation to health and diseases.

Obesity is accompanied by FGF21 resistance, both in the periphery and in the brain, due to receptor downregulation and reduced FGF21 transfer to cerebrospinal fluid ${ }^{43-45}$. Central FGF21 resistance may be among the overlooked underlying pathologies contributing to aberrant dietary choices that exacerbate obesity and diabetes. Of interest, hepatic SIRT1 promotes FGF21 transcription ${ }^{46}$. Thus, systemic activation of SIRT1 may promote systemic FGF21 signalling by increasing the endocrine 

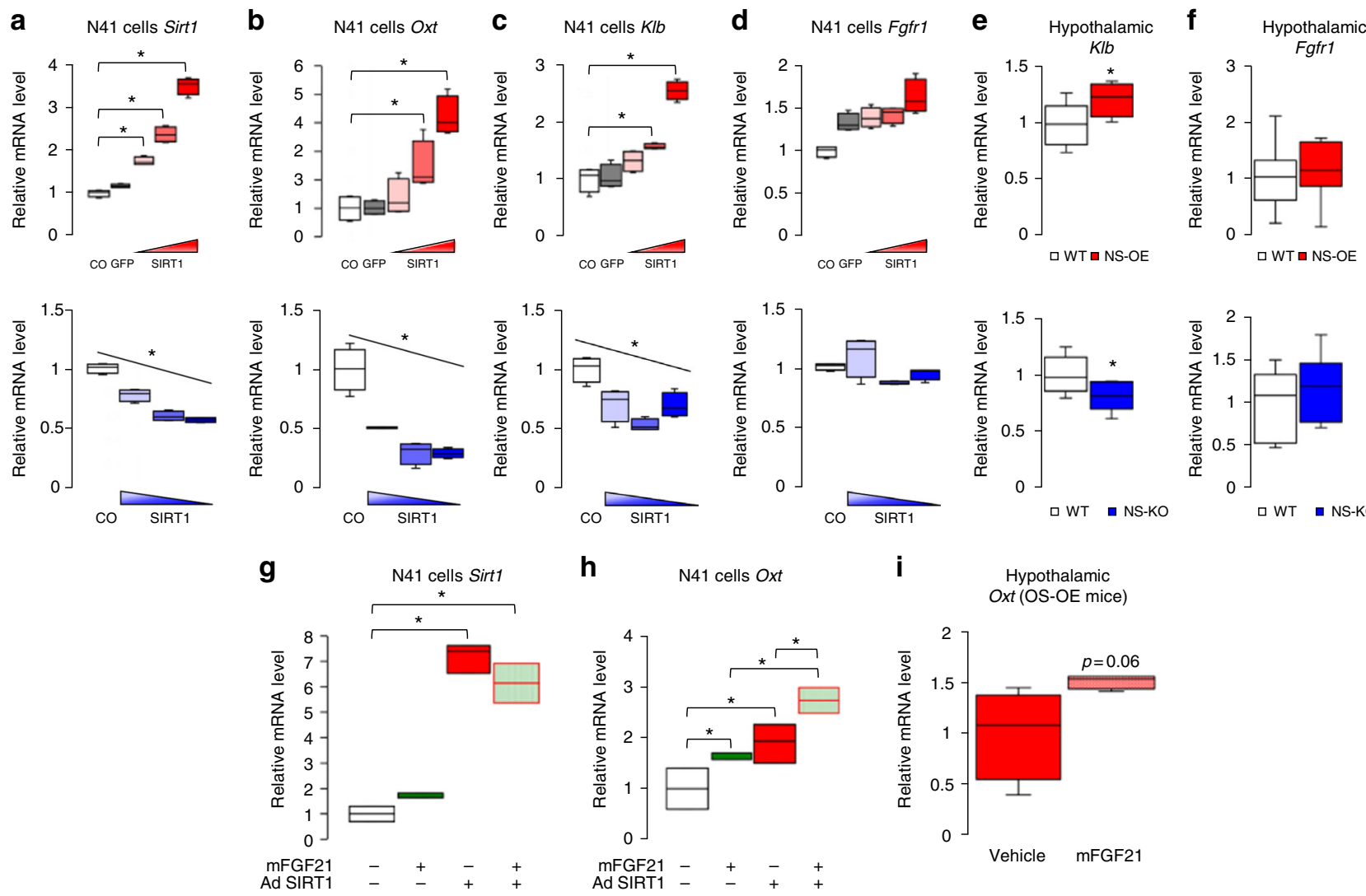

h
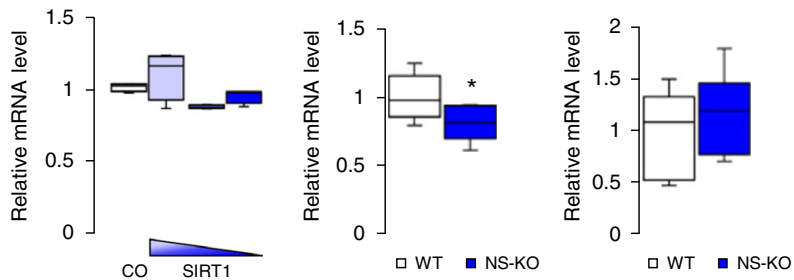

\section{i}

Hypothalamic Oxt (OS-OE mice)
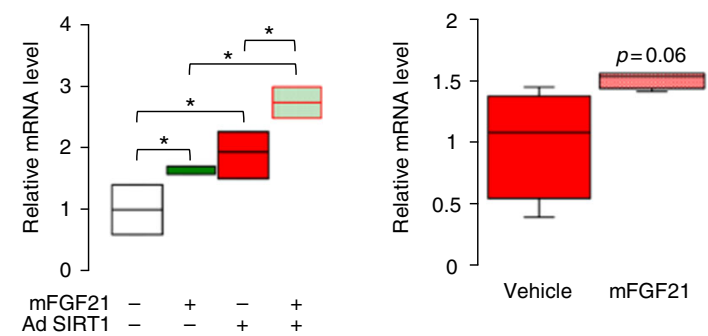

Vehicle mFGF21
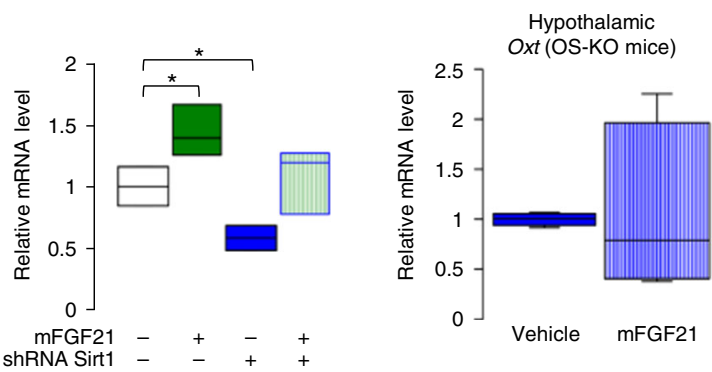

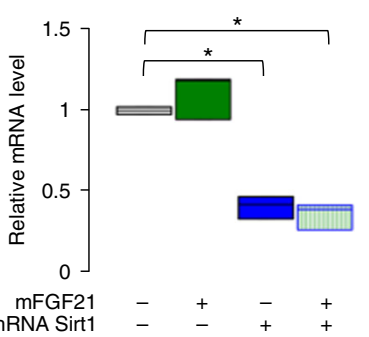

j

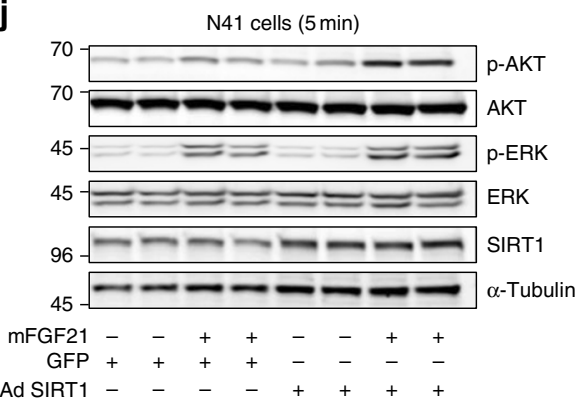

$\mathbf{k}$

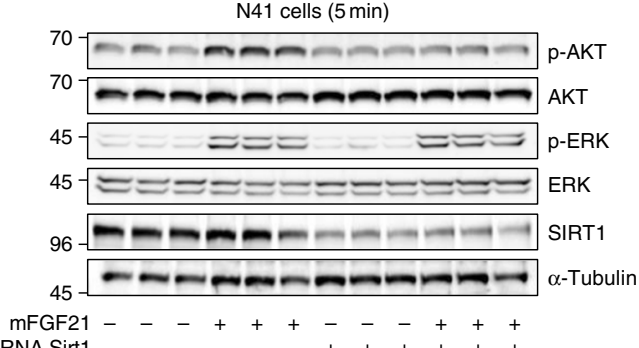

Fig. 7 SIRT1 enhances KLB-AKT signalling and promotes FGF21-induced Oxt expression. a-d Effects of Sirt1 manipulation on gene expression in hypothalamic N41 cells. Sirt1 expression was manipulated by viral vectors to different degrees. Sirt1 expression in untreated (CO), control (GFP) and viral infected cells (red, Sirt1 overexpressing; blue, Sirt1 silencing). Shading indicates low (light) to high (dark) MOls or knockdown efficiencies. Manipulating Sirt1 expression (a) affected expression of Oxt (b) and Klb (c), but not Fgfrl (d) ( $n=4$ per group). e, f Hypothalamic KIb and Fgfr 1 mRNA expression in WT, NSOE (red) and NS-KO (blue) mice ( $n=6$ per group). $\mathbf{g}$, h Effects of Sirt1 overexpression (top) and Sirt1 silencing (bottom) on FGF21-induced Oxt expression. N41 cells were treated with or without 100 nM mFGF21 for 8 h; expression levels of Sirt1 (g) and Oxt (h) ( $n=3$ per group). i Hypothalamic Oxt mRNA expression levels in to OS-OE and OS-KO mice after IP injection of mFGF21 ( $1 \mathrm{mg} / \mathrm{kg}$ ) or water (vehicle) ( $n=4$ per group). j, $\mathbf{k}$ Effects of overexpressing (j) ( $n=2$ per group) or silencing ( $(\mathbf{k})$ ( $n=3$ per group) Sirt1 expression on FGF21-induced intracellular signalling analysed by western blotting. After viral infection, N41 cells were incubated for 5 min in the absence or presence of 100 nM mFGF21. Data are shown as box and whisker plots (centre line, median; box limits, upper and lower quartiles; whiskers, the minimum and maximum value of a data set). ${ }^{*} p<0.05$. Data in $\mathbf{a}-\mathbf{h}$ were replicated more than three times, and data in $\mathbf{j}$, $\mathbf{k}$ were replicated twice in the laboratory. See also Supplementary Fig. 6 


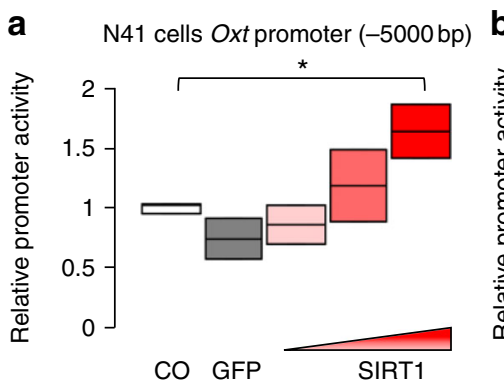

d

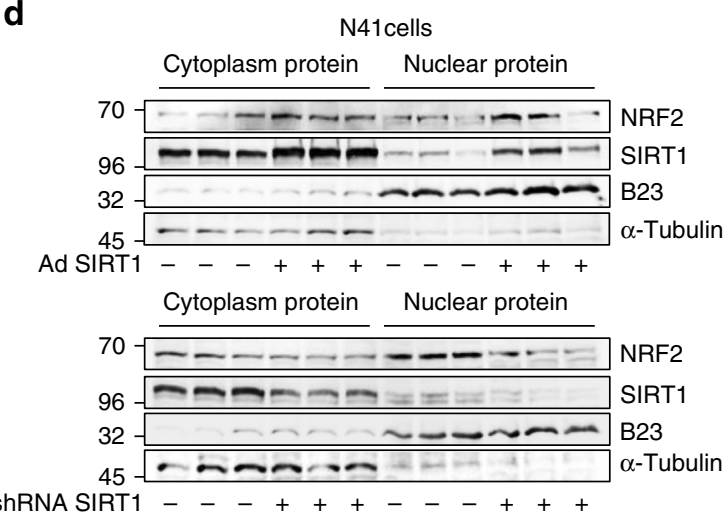

h

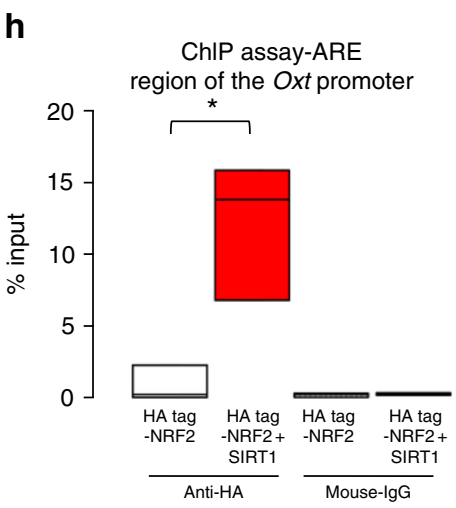

ShRNA SIRT1 - - - + + + - - + +
N41 cells Oxt promoter

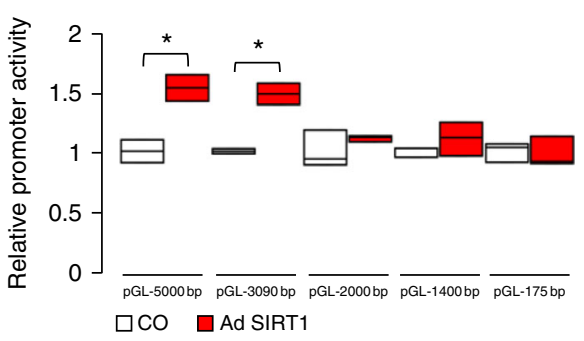

e

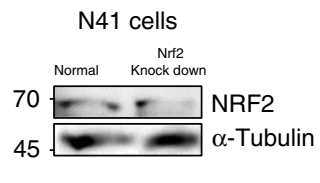

c

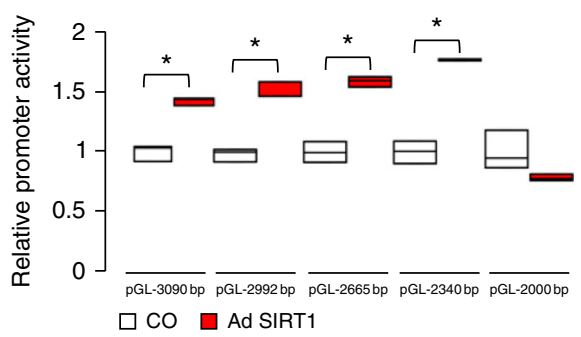

f

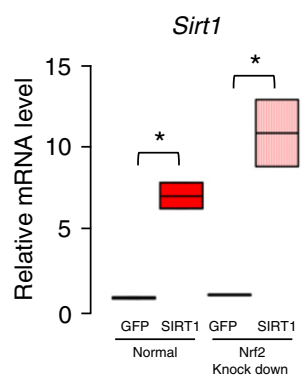

g

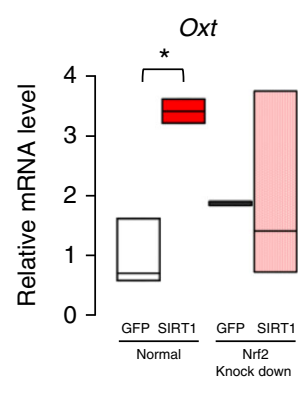

i
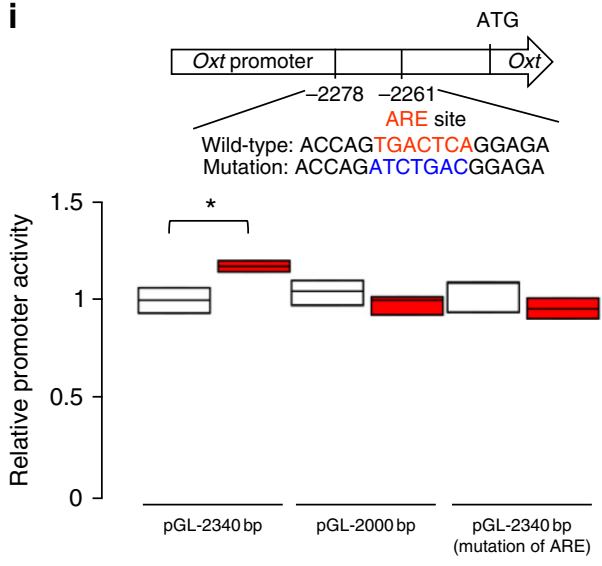

$\square \mathrm{CO} \quad \square$ Ad SIRT1

Fig. 8 SIRT1 regulates expression of Oxt through NRF2. a-c Luciferase assays of Oxt promoters of various lengths in N41 cells. Luciferase activity was measured in response to adenoviral overexpression of Sirt1 (ad-Sirt1), the control virus (GFP) and uninfected cells (co, normalized to 1). Sirt1 activation of full-length ( -5000$)$ (a) and truncated (b, c) Oxt promoters ( $n=3$ per each group). d The effects of overexpressing (upper panels) or silencing (lower panels) Sirt1 expression in N41 cells on nuclear NRF2 protein levels. Immunoblots of nuclear and cytoplasmic fractions probed for NRF2, SIRT1, B23 (nuclear marker) and $\alpha$-tubulin (cytoplasm marker) ( $n=3$ per group). e-g Effect of Nrf2 knockdown on SIRT1-induced Oxt expression. After lentiviral knockdown of Nrf2 in N41 cells (e), Sirt1 expression levels were manipulated with adenoviral overexpression (f), and Oxt expression was measured (g) ( $n=4$ per group). h ChIP performed on HA-tagged NRF2, FLAG-tagged SIRT1 or pCMV5 (empty)-transfected N41 cells using HA tag or mouse IgG antibody. ChIP DNA was subjected to quantitative PCR using primers specific for the ARE region in the Oxt promoter. Data are presented relative to input ( $n=3$ per group). i Activity of the -2430 -bp promoter with and without mutations in the ARE region ( $n=3$ per group). Data are shown as box and whisker plots (centre line, median; box limits, upper and lower quartiles; whiskers, the minimum and maximum value of a data set). ${ }^{\star} p<0.05$. Data in $\mathbf{a}-\mathbf{i}$ were replicated twice in the laboratory. See also Supplementary Fig. 7

supply of the ligand (FGF21) from the liver and increasing expression of the co-receptor ( $\beta$-klotho) in the target organs. These changes would lead to enhanced intracellular signalling in FGF21 target cells, such as in Oxt neurons, as shown in this work. Obese people and patients with diabetes have decreased circulating Oxt levels ${ }^{47}$, and intranasal Oxt treatment causes a shift in metabolism from using carbohydrates to using fat ${ }^{48}$. Based on our finding that central FGF21 signalling stimulates Oxt neurons and suppresses simple sugar preference, we speculate that improving central FGF21 sensitivity could improve diet selection to match the peripheral tissue substrate need through Oxt and improve health in obese and diabetic people. Furthermore, this improvement could elevate stress resilience by promoting Oxt action because Oxt ameliorates the stress response ${ }^{49}$.

We also found that multiple longevity genes co-ordinately regulated the simple sugar preference in mice. Both FGF21 and NRF2 are longevity genes that extend the lifespan in model organisms ${ }^{50-53}$. SIRT1 may promote a long lifespan in part by coordinating multiple longevity pathways, including previously described targets FOXO $^{54}$ and $\mathrm{HSF}^{55}$. Furthermore, Oxt 
a

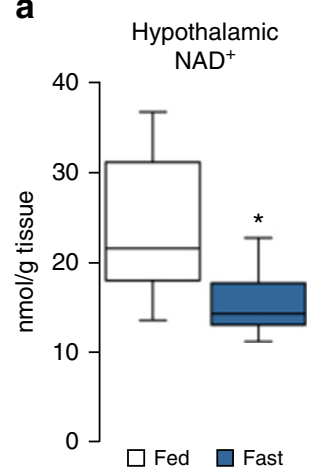

b
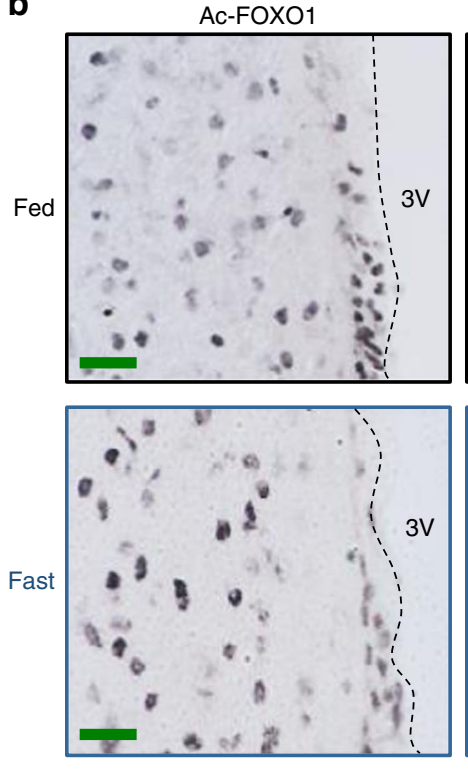

d

C

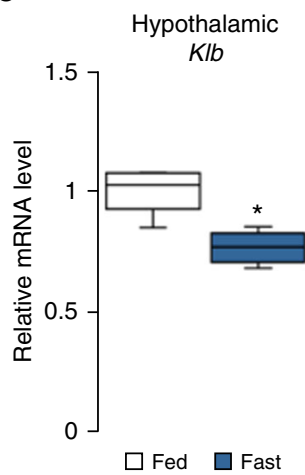

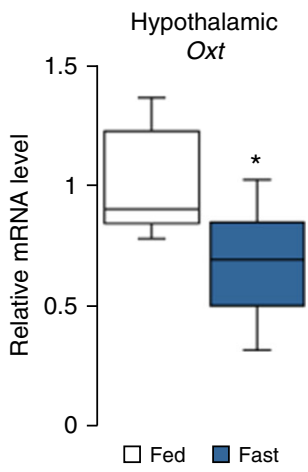

e

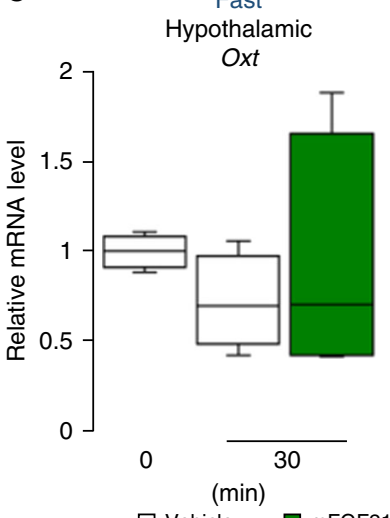

Ac-p53
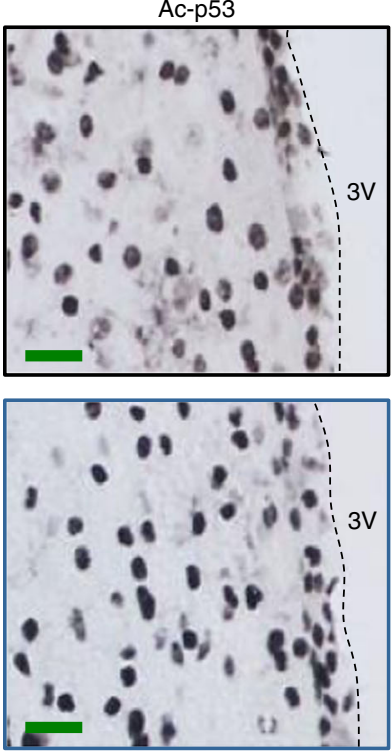

f

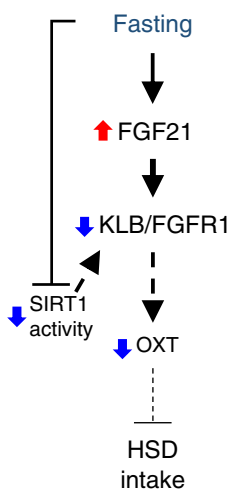

Ac-NF-kB
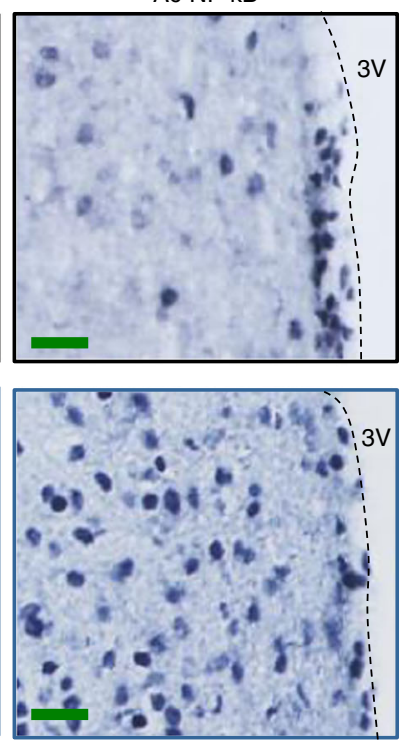

$\square$ Vehicle

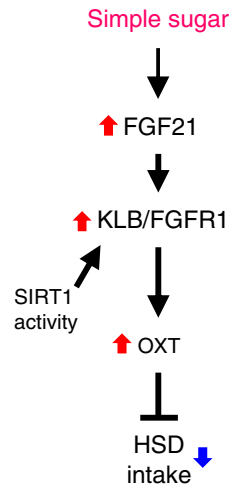

Fig. 9 Fasting attenuates hypothalamic SIRT1 activity and hypothalamic FGF21-Oxt signalling. a The effects of feeding and fasting on hypothalamic NAD ${ }^{+}$ levels ( $n=12$ in the Fed group, $n=13$ in the Fasted group). b Photomicrographs depict hypothalamus coronal section from wild-type C57BL/6 mice (fed or fasted state) that received 3rd ICV injection of $0.5 \mu \mathrm{l}$ of TSA $(10 \mu \mathrm{g} / \mu \mathrm{l})$. The length of scale bars is $100 \mu \mathrm{m}$. The sections were immunostained to identify Ac-FOXO1, Ac-p53 and Ac-NF-KB protein (black or blue) expression. c, d The effects of feeding and fasting on hypothalamic expression levels of Klb (c) ( $n=6$ in the Fed group, $n=7$ in the Fasted group) and Oxt (d) ( $n=6$ in the Fed group, $n=7$ in the Fasted group) in C57BL6/J mice fed ad libitum or fasted for $24 \mathrm{~h}$. e Hypothalamic Oxt mRNA expression levels after IP injection of mFGF21 (1 mg/kg) or water (vehicle) in 24-h-fasted C57BL6/J mice ( $n=$ 4 per group). f Schematic diagrams of hypothalamic FGF21-Oxt signalling and SIRT1 in fasting and feeding. (Left) Fasting increased circulating FGF21 levels but suppressed hypothalamic SIRT1 activity, which was associated with the down-regulation of FGF21-KLB-OXT signalling. (Right) During simple sugar feeding, hypothalamic SIRT1 activity was maintained, and FGF21 functioned as a metabolic signal for regulating the simple sugar preference through the FGF21-OXT signalling. Data are shown as box and whisker plots (centre line, median; box limits, upper and lower quartiles; whiskers, the minimum and maximum value of a data set). ${ }^{\star} p<0.05$. 3V, 3rd ventricle; Ac. acetylated. See also Supplementary Fig. 8

attenuates stress responses ${ }^{49}$. Metabolic shifts, altered diet selection and improved stress resilience may represent an integrated biological strategy for survival against physical and psychological stressors. Other evolutionarily conserved energy sensor molecules that can cause a metabolic switch between glycolysis and fatty acid oxidation in peripheral tissues may work in the nervous system in a similar manner.

How some neuropeptides regulate feeding behaviour in a macronutrient-specific manner remains elusive. One potential mechanism is that release of such neuropeptides may be triggered only by the macronutrient-specific signals, such as FGF21 regulating Oxt, as shown here. Another potential mechanism is that neuropeptides may regulate particular projection target(s) in a macronutrient-specific manner. For instance, Oxt neurons project to multiple sites within the reward system, including the NAc, amygdala and $\mathrm{VTA}^{22}$. Oxt projections to $\mathrm{NAc}^{56}$ and $\mathrm{VTA}^{57}$ have been implicated in the regulation of sucrose preference, and the VTA projection gates social reward ${ }^{58}$. Oxt neurons also project to other brain areas; intranasal administration of Oxt in humans suppresses hypothalamic activation to visual food cues ${ }^{59}$, and enhanced cognitive control in a food craving task accompanied by neural activation of the prefrontal cortex, which is involved in inhibitory control ${ }^{60}$. Because only a fraction of PVH Oxt neurons responded to FGF21 in the current work, analysing the projection sites of FGF21-responsive PVH Oxt neurons may improve understanding of how Oxt controls simple sugar preference at the neurocircuit level. Indeed, Oxt neural projections might selectively reduce the hedonic value of sugars.

Here we identified the link between FGF21 and Oxt by focusing on the metabolic signals that are trigged by simple sugar 


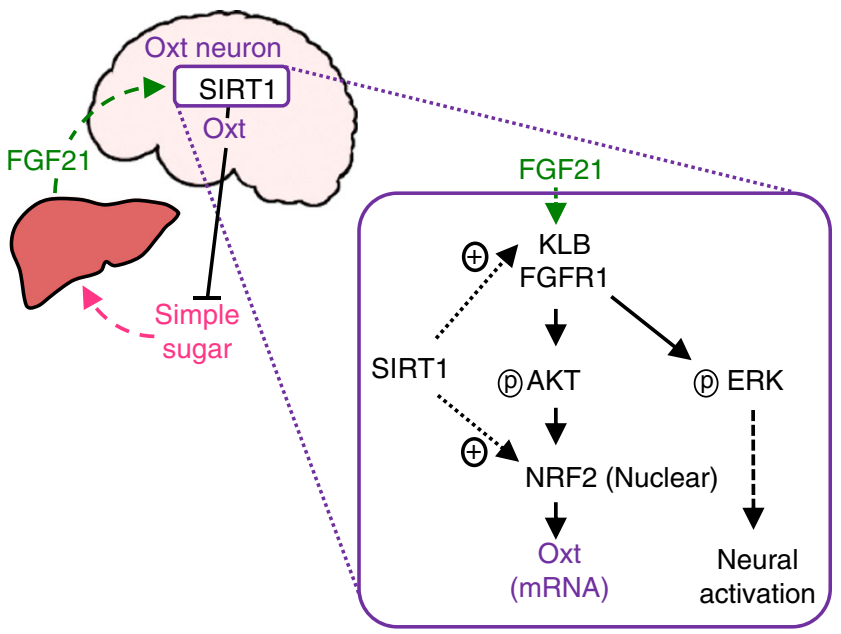

Fig. 10 Proposed model of simple sugar preference regulation by neuronal SIRT1. Ingestion of simple sugar promotes secretion of FGF21 from the liver. The hepatokine FGF21 acts as an endocrine signal to Oxt neurons and promotes Oxt transcription (AKT signalling) and neuronal activation (ERK signalling). Oxt suppresses simple sugar preference by acting on OXTR. SIRT1 promotes FGF21 sensitivity in Oxt neurons and stimulates Oxt transcription through NRF2, thereby potentiating the negative feedback regulation of simple sugar preference through FGF21 and Oxt

ingestion. We aimed to address the role of taste versus metabolic signals (post-ingestive effect) by using non-nutritive artificial sweeteners. When we performed the 2-bottle tests in sugar-naive mice, we did not observe any differences in the preferences for the sweeteners in both OS-OE and OS-KO mice (Fig. 4f, g). However, when the same 2-bottle tests were performed with mice that had experience sugar previously, we found that the preference for saccharin was similarly affected in both OS-OE and OS-KO mice (Supplementary fig. 9). It has been known that non-nutritive artificial sweeteners could have post-ingestive metabolic effects called cephalic phase responses ${ }^{61}$. It is a form of conditioningbased learning, in which the taste cue (sweet taste) experience upon sugar ingestion become associated with the post-ingestive metabolic experience, so that anticipatory pre-absorptive metabolic responses (such as salivation, gastric acid secretion, secretion of metabolic hormones, and thermogenesis) are induced by the conditioned taste cue ${ }^{30,31}$. Therefore, performing two-bottle experiments in sugar-naive mice was important for addressing the role of taste versus metabolic signals in our cases. Although both FGF21 and Oxt had been implicated in the preferences to artificial sweeteners without affecting taste reactivity $24,32,34,62,63$, it is not clear if these past works were performed in sugar-naive animals. Pharmacological administration of recombinant FGF21 or FGF21 analogue decreased preferences to sugars and sweeteners $^{32,34}$. However, Fgf $21 \mathrm{Tg}$ mice showed decreased preferences to both sugar and saccharin ${ }^{34}$, while Fgf $21 \mathrm{KO}$ mice showed increased preference to sugars but not saccharin ${ }^{32}$. Another possible explanation for the differences in the sweetener preferences between our sugar-naive mice and the past literature is microbiome. The artificial sweeteners altered microbiome and disturbed systemic glucose homoeostasis ${ }^{64,65}$, and Lactobacillus reuteri increased $\mathrm{PVH}$ oxytocin in mice ${ }^{66}$, suggesting that the preference for the artificial sweeteners could be regulated through microbiome and Oxt. The differences in microbiome between our sugar-naive mice and mice used in the past literature may have caused the differences in the preferences to sweeteners. Therefore, the mechanisms that regulate preferences to sweeteners remain elusive.
This study also did not resolve the molecular mechanisms underlying the regulation of fat preference by SIRT1. At the least, Oxt is not responsible for the regulation of fat preference in NSOE and NE-KO mice because SIRT1 manipulation in Oxt neurons (OS-OE and OS-KO mice) did not affect fat preference (Fig. 3f). A potential mechanism might operate by improving the sensitivity to metabolic signals because fat intake drives a positive feedback loop ${ }^{21}$ and SIRT1 improves central sensitivity to hormones such as FGF21 and leptin ${ }^{10,11}$. Furthermore, different fatty acids have different effects on health; thus, SIRT1 may modulate preferences for particular types of fatty acids. Indeed, we found that SIRT1 modulated only the preference for simple sugars, not all carbohydrates. Our understanding of homoeostatic diet selection therefore might be advanced by studies that focus on identifying fatty acid preferences under SIRT1 regulation.

In summary, our investigation of the mechanisms that regulate macronutrient-based diet selection unexpectedly revealed that Oxt is regulated by SIRT1, FGF21 and NRF2. Understanding the homoeostatic mechanisms for macronutrient selection may unveil an underlying pathophysiology of aberrant diet selection in obesity and lead to the development of interventions that promote healthy diet choice.

\section{Methods}

Animals. Mice were generated to overexpress neuron-specific Sirt1 (NS-OE) and Oxt-neuron-specific Sirt1 (OS-OE). These strains were generated by breeding mice that harboured Rosa26-Sirt $1^{10}$ with mice that harboured neuron-specific Tau-Cre ${ }^{15}$ (for NS-OE) or Oxt-neuron-specific Oxt-ires-Cre ${ }^{28}$ (for OS-OE). Mice were also generated to knock out neuron-specific Sirt1 (NS-KO) and Oxt-neuron-specific Sirt1 (OS-KO). These strains were generated by breeding mice that harboured a floxed Sirt 1 allele ${ }^{16}$ with mice harbouring either Tau-Cre recombinase (NS-KO) or Oxt-ires-Cre (OS-KO) recombinase. Rosa26-Sirt1 mice had a 129/Bl6 mixed background, maintained with three backcrosses to Bl6. Oxt-ires-Cre transgenic mice had a 129/Bl6 mixed background, maintained with five backcrosses to Bl6. Tau-Cre and Sirt1-flox mice had a pure C57BL6/J genetic background.

All animals were maintained in a specific pathogen-free space under a $12-\mathrm{h}$ light/dark regimen. At the time of the experiments, mice were 10-16 weeks old. Unless otherwise specified, male littermates were randomly assigned to all experimental groups. The NC (CE-2; CREA Japan, Tokyo, Japan), HSD (F2HScD; Oriental Yeast Co. Ltd, Tokyo, Japan), HFD (HFD32; Oriental Yeast Co. Ltd, Tokyo, Japan), and water were provided ad libitum. Intakes of NC, HSD and HFD and body weight were measured daily after 11 weeks of age. None of the animals included in the data analyses displayed any health impairments.

All experimental procedures were performed in accordance with the Guide for the Care and Use of Laboratory Animals of the Science Council of Japan and approved by the Animal Experiment Committee of Gunma University.

Two-food choice studies. For the two-food choice studies, 10-week-old male or female mice with wild-type, NS-OE, NS-KO, OS-OE or OS-KO genotypes were acclimated for 1 week to feeding from multifeeders (Shinfactory, Fukuoka, Japan). Then, mice were given access to a multifeeder that provided either NC vs. HSD or $\mathrm{NC}$ vs. HFD. Food intake and body weight were measured daily for 5 days $(n=6$ per group). The positions of the two food choices were switched every day to exclude positional effects.

Long and brief two-bottle choice studies. For the two-bottle choice studies, 10 week-old male mice with wild-type, NS-OE, NS-KO, OS-OE or OS-KO genotypes were first acclimated to cages with two bottles (Drink-O-Meter) that contained water for 1 week. For the long two-bottle choice studies, mice were given ad libitum access to one bottle with water and one bottle with a test solution for 3 days $(n=7$ in the WT group; $n=6$ in the OS-OE and OS-KO groups). The test solution contained one of the following: $100 \mathrm{mM}$ glucose (Wako, Osaka, Japan), $100 \mathrm{mM}$ fructose (Wako, Osaka, Japan), $100 \mathrm{mM}$ sucrose (Nakarai, Kyoto, Japan), 2\% dextrin (Nakarai, Kyoto, Japan), 0.2\% saccharin (Wako, Osaka, Japan), 0.2\% aspartame (Tokyo Chemical Industry, Tokyo, Japan) or $1.5 \mathrm{mM}$ quinine (Wako, Osaka, Japan). NC was also available ad libitum throughout the test period. The positions of the two bottles were changed every day to exclude positional effects.

For the brief two-bottle choice studies, mice were given ad libitum access to one bottle with water and one bottle with $100 \mathrm{mM}$ sucrose or $0.2 \%$ saccharin solution for only $10 \mathrm{~min}$ ( $n=6$ per group). NC was also available ad libitum throughout the test period. The positions of the two bottles were changed every 3 min to exclude positional effects. 
Effect of Oxtr antagonist on sucrose selection behaviour. Eleven-week-old male mice with wild-type, NS-OE and OS-OE genotypes were injected IP with the Oxtr antagonist OXTR-A (L-368, 899, $5 \mathrm{mg} / \mathrm{kg}$, twice a day for 3 days; Tocris Bioscience, Bristol, UK) or water (vehicle). After the OXTR-A treatment was initiated, mice were presented with two food choices (NC vs. HSD) in multifeeders. Food intake was measured daily for 3 days ( $n=5$ per group for wild-type vs. NS-OE; $n=6$ per group for wild-type vs. OS-OE). The positions of the two foods in the multifeeders were changed every day to exclude positional effects.

Indirect calorimetry and locomotor activity measurement. Oxygen consumption and $\mathrm{CO}_{2}$ production were measured in individual male OS-KO mice (control, $n=6$; KO, $n=6$ ) and OS-OE mice (control, $n=6$; OE, $n=7$ ) at 8 weeks of age, with an Oxymax apparatus (Columbus Instruments, Columbus, $\mathrm{OH}$, USA). The $\mathrm{O}_{2}$ and $\mathrm{CO}_{2}$ measurements were performed every $18 \mathrm{~min}$ for each mouse over a 3-day period and the data from the final day were analysed. Locomotor activity was measured with the ACTIMO-100 (Shinfactory).

Generation of adeno-associated viral (AAV) vector. AAV Helper-Free System (Agilent Technologies, Inc., Santa Clara, CA, USA) were used for the generation of the AAV2-hSyn-FLEX-mCherry or AAV2-hSyn-FLEX-hM4Di-mCherry viral vector. Purification method was modified from the previously published protocol ${ }^{67}$. Briefly, HEK293 cells were transfected with a pAAV-hSyn-FLEX-mCherry or pAAV-hSyn-FLEX-hM4Di-mCherry vector plasmid (gifts from Dr. Bryan Roth at the University of North Carolina, USA), pHelper and pAAV-RC2 (purchased from Cell Biolabs Inc, San Diego, CA, USA) using a standard calcium phosphate method. Three days after transfection, cells were collected and suspended in artificial CSF solution containing (in mM) $124 \mathrm{NaCl}, 3 \mathrm{KCl}, 26 \mathrm{NaHCO}_{3}, 2 \mathrm{CaCl}_{2}, 1$ $\mathrm{MgSO}_{4}, 1.25 \mathrm{KH}_{2} \mathrm{PO}_{4}$ and $10 \mathrm{D}$-Glucose. Following four freeze-thaw cycles, the cell lysates were treated with Benzonase nuclease (Merck, Darmstadt, Germany) at $45^{\circ} \mathrm{C}$ for $15 \mathrm{~min}$, then centrifuged twice at $20,000 \times \mathrm{g}$ for $10 \mathrm{~min}$ at $4^{\circ} \mathrm{C}$. The supernatant was used as the virus-containing solution. Quantitative PCR was performed to measure the titre of purified virus. Virus aliquots were then stored at $-80^{\circ} \mathrm{C}$ until use for experiment.

DREADD experiment. AAV2-hSyn-FLEX-mCherry or AAV2-hSyn-FLEXhM4Di-mCherry virus was injected into and specifically expressed in PVH Oxt neurons. The skulls of 10-week-old male OS-OE mice were exposed and a small hole drilled above each side of the PVH. Bilateral injection was performed with a Hamilton $10-\mu$ l syringe (Sigma Aldrich, St Louis, MO, USA) filled with $1 \mu$ lof virus $\left(9.94 \times 10^{11}\right.$ copies $\left./ \mathrm{ml}\right)$ at the following coordinates: bregma: AP: $-0.82 \mathrm{~mm}$; DV: $-4.75 \mathrm{~mm}$; and RL: $\pm 0.25 \mathrm{~mm}$. The injection speed was controlled at $100 \mathrm{nl} / \mathrm{min}$ with a micromanipulator (IMS20, Narishige, Tokyo, Japan). Experiments were performed 2 weeks post-injection to allow for recovery and viral expression.

The AAV2-hSyn-FLEX-mCherry virus-or AAV2-hSyn-FLEX-hM4DimCherry-expressing mice were injected IP with clozapine $(1 \mu \mathrm{g} / \mathrm{kg}$ ) or PBS $(\mathrm{pH} 7.4$, vehicle) once a day at 13:00, and mice then were given access to a multifeeder that provided either NC vs. HSD. Food intake was measured daily for 3 days $(n=8$ per group). The positions of the two food choices were switched every day to exclude positional effects. We used the injection of clozapine instead of clozapine-N-oxide (CNO), because upon systemic CNO injections, converted clozapine readily enters the brain and occupies central nervous system-expressed DREADD receptors; in contrast, systemic subthreshold clozapine injections induce preferential DREADDreceptor-mediated behaviours ${ }^{29}$.

After the completion of study period, mice were anesthetized, and then perfused transcardially, first with PBS (pH 7.4), then with $4 \%$ paraformaldehyde (PFA) Dissected brains were post-fixed in a $4 \%$ PFA solution at $4{ }^{\circ} \mathrm{C}$ overnight, then incubated in a $20 \%$ sucrose solution at $4{ }^{\circ} \mathrm{C}$ overnight. Brains were sectioned coronally, at a thickness of $25 \mu \mathrm{m}$. Sections were collected in PBS, pH 7.4, transferred to a cryoprotectant solution, and stored at $-20^{\circ} \mathrm{C}$.

For immunostaining, sections were incubated with $3 \%$ normal donkey serum (NDS) in PBS containing 0.3\% Triton X-100 (3\% NDS/0.3\% PBT) for $1 \mathrm{~h}$. Then we added rabbit anti-Oxt antibody (1:800; \#T-4087.0050, Peninsula Laboratories, San Carlos, CA, USA) and rat anti-mCherry antibody (1:600; \#16D7, Thermo Fisher, USA) diluted in $3 \% \mathrm{NDS} / \mathrm{PBT}$, and sections were incubated overnight at $4{ }^{\circ} \mathrm{C}$. After washing in PBS that contained $0.3 \%$ Triton X-100, sections were incubated for $1 \mathrm{~h}$ with Alexa Fluor 488 Donkey anti-rabbit IgG (1:400; R37118, Thermo Fisher Scientific, Waltham, MA, USA) and rat anti-Cy3 antibodies (1:200; Jackson ImmunoReseach, West Grove, PA, USA) diluted in 3\% NDS/0.3\% PBT. Fluorescence images were acquired with a BZ-9000 fluorescence microscope (KEYENCE, Osaka, Japan).

Double staining immunohistochemistry. For c-Fos/Oxt double-staining, 11week-old male C57BL/6 mice were injected IP with murine FGF21 (mFGF21; 1 $\mathrm{mg} / \mathrm{kg}$; BioVendor, Modrice, Czech Republic) or water (vehicle) ( $n=4$ per group). At $2 \mathrm{~h}$ after the injection, mice were anaesthetized with sodium pentobarbital $(50 \mathrm{mg} / \mathrm{kg}, \mathrm{IP})$ and perfused transcardially with ice-cold $0.05 \mathrm{M}$ PBS (pH 7.4) followed by a fixation solution containing 4\% PFA. Dissected brains were postfixed in $4 \%$ PFA solution at $4{ }^{\circ} \mathrm{C}$ overnight, then incubated in $20 \%$ sucrose solution at $4^{\circ} \mathrm{C}$ overnight. Brains were sectioned coronally, at a thickness of $25 \mu \mathrm{m}$, and stored in cryoprotectant solution at $-30^{\circ} \mathrm{C}$ until use. We performed double immunostaining of c-Fos and Oxt as follows. Sections were washed in PBS, then treated with $0.3 \% \mathrm{H}_{2} \mathrm{O}_{2}$ diluted in PBS for $15 \mathrm{~min}$. Sections were blocked with $3 \%$ NDS diluted in PBS containing $0.25 \%$ Triton X-100 for $30 \mathrm{~min}$. The sections were incubated in rabbit anti-c-Fos antiserum (1:25,000; \#ABE457, Merck Millipore, Billerica, USA) diluted in blocking solution overnight at $22-25^{\circ} \mathrm{C}$. After being washed in PBS, the sections were incubated with biotinylated goat anti-rabbit IgG (1:400; BA-1000, Vector Laboratories, Burlingame, CA, USA) for $40 \mathrm{~min}$ and incubated with $A B C$ reagent (Vector Laboratories) for $40 \mathrm{~min}$. The sections were washed in PBS and $0.175 \mathrm{M}$ sodium acetate buffer ( $\mathrm{pH}$ 5.6), and colour was developed with a nickel-diaminobenzidine solution $(10 \mathrm{~g} / \mathrm{l}$ nickel ammonium sulphate, $0.2 \mathrm{~g} / \mathrm{l}$ diaminobenzidine and $0.006 \% \mathrm{H}_{2} \mathrm{O}_{2}$ in sodium acetate buffer). After being washed in $\mathrm{PBS}$, the sections were treated with $0.3 \% \mathrm{H}_{2} \mathrm{O}_{2}$ diluted in PBS for $15 \mathrm{~min}$ and blocked with blocking solution. Incubation followed in rabbit anti-Oxt antibody $(1: 1200)$ diluted in blocking solution overnight at $22-25^{\circ} \mathrm{C}$. After another wash in PBS, the sections were incubated with biotinylated goat anti-rabbit IgG (1:400) for $40 \mathrm{~min}$ and incubated with $\mathrm{ABC}$ reagent (Vector Laboratories) for 40 min. After a final wash in PBS and $0.1 \mathrm{mM}$ Tris- $\mathrm{HCl}$ buffer $(\mathrm{pH}$ 7.5) containing $0.15 \mathrm{mM}$, colour was developed with a diaminobenzidine solution.

For Oxt/SIRT1 double staining of WT and OS-OE mouse brain sections, the sections were incubated with 3\% NDS in PBS containing 0.1\% Triton X-100 (3\% NDS/0.1\% PBT) for $1 \mathrm{~h}$ and then overnight at $4{ }^{\circ} \mathrm{C}$ with rabbit anti-OXT antibody (1:800) and mouse anti-SIRT1 antibody (1:200; \#ab110304, Abcam, Cambridge, UK) diluted in $3 \% \mathrm{NDS} / 0.1 \%$ PBT. After washing in $0.1 \% \mathrm{PBT}$, sections were incubated for $1 \mathrm{~h}$ with Alexa Fluor 488 Donkey anti-Rabbit IgG (1:400) and Alexa Fluor 594 Donkey Anti-Mouse IgG (1:400; R37115, Thermo Fisher Scientific) diluted in 3\% NDS/0.1\% PBT. Fluorescence images were acquired using BZ-9000 (KEYENCE, Osaka, Japan).

For Oxt/SIRT1 double staining of WT and OS-KO mouse brain sections, the sections were washed in PBS, and then treated with $3 \% \mathrm{H}_{2} \mathrm{O}_{2}$ diluted in PBS for $5 \mathrm{~min}$. Sections were blocked with $3 \% \mathrm{NDS} / 0.1 \%$ PBT for $30 \mathrm{~min}$. The sections were incubated in mouse anti-SIRT1 antibody (1:1000) diluted in blocking solution overnight at $22-25^{\circ} \mathrm{C}$. After washing in PBS, the sections were incubated with labelled polymer-HRP (K4061, Agilent Technologies, California, USA) for $30 \mathrm{~min}$. The sections were washed in PBS and color was developed with a nickeldiaminobenzidine solution $(10 \mathrm{~g} / \mathrm{l}$ nickel ammonium sulphate, DAB substrate kits (K3468, Agilent Technologies, California, USA)). After washing in PBS, the sections were treated with $3 \% \mathrm{H}_{2} \mathrm{O}_{2}$ diluted in PBS for $5 \mathrm{~min}$. The sections were blocked with blocking solution. The section were incubated in rabbit anti-OXT (1:800) diluted in blocking solution overnight at $22-25^{\circ} \mathrm{C}$. After washing in PBS, the sections were incubated with labelled polymer-HRP for $30 \mathrm{~min}$. The sections were washing in PBS and color was developed with DAB substrate kits.

Evaluation of the acetylation levels of SIRT1 substrates. In order to detect the difference in acetylation status of SIRT1 substrates in mice, they were anesthetized and $0.5 \mu \mathrm{l}$ of trichostatin $\mathrm{A}(10 \mu \mathrm{g} / \mu \mathrm{l})$ was injected into the third ventricle at the following coordinates: bregma: AP:-1.70 mm; DV: $-5.3 \mathrm{~mm}$; and RL:0.00 mm. At $1 \mathrm{~h}$ after the injection, mice were anesthetized with sodium pentobarbital $(50 \mathrm{mg} /$ $\mathrm{kg}, \mathrm{IP}$ ) and perfused transcardially with ice-cold $0.05 \mathrm{M} \mathrm{PBS}$ ( $\mathrm{pH}$ 7.4) followed by a fixation solution containing 4\% paraformaldehyde (PFA). Dissected brains were post-fixed in $4 \%$ PFA solution at $4{ }^{\circ} \mathrm{C}$ overnight, then they were incubated in $20 \%$ sucrose solution at $4{ }^{\circ} \mathrm{C}$ overnight. Brains were sectioned coronally, at a thickness of $25 \mu \mathrm{m}$, and stored in cryoprotectant solution at $-30^{\circ} \mathrm{C}$ until use.

For Ac-FOXO1, Ac-p53 and Ac-NF-kB immunostaining of WT, NS-OE and C57BL/6 (fed NC, HSD or HFD; and fed or fasted state) mouse brain sections, the sections were washed in PBS, and then treated with $3 \% \mathrm{H}_{2} \mathrm{O}_{2}$ diluted in PBS for $5 \mathrm{~min}$. Sections were blocked with $3 \% \mathrm{NDS} / 0.1 \%$ PBT for $30 \mathrm{~min}$. The sections were incubated in rabbit anti-Ac-FOXO1 antibody (1:250; \#sc-49437-R, Santa Cruz Biotechnology), rabbit anti-Ac-p53 antibody (1:150; \#2525, Cell Signaling) and rabbit anti-Ac-NF- $\kappa B$ antibody (1:400; \#19870, Abcam) diluted in blocking solution overnight at $22-25^{\circ} \mathrm{C}$. After washing in PBS, the sections were incubated with labelled polymer-HRP (K4061, Agilent Technologies) for $30 \mathrm{~min}$. The sections were washed in PBS and color was developed with a nickel-diaminobenzidine solution.

For Oxt/Ac-FOXO1, Ac-p53 and Ac-NF-kB double staining of WT and OS-OE mouse brain sections, the sections underwent the same procedures as described above. After the development of the acetyl substrate signals, the sections underwent the following additional steps for Oxt staining. After washing in PBS, the sections were treated with $3 \% \mathrm{H}_{2} \mathrm{O}_{2}$ diluted in PBS for 5 min. The sections were blocked with blocking solution. The section were incubated in rabbit anti-OXT (1:1,000; \#T-4087.0050, Peninsula Laboratories, San Carlos, CA, USA) diluted in blocking solution overnight at $22-25^{\circ} \mathrm{C}$. After washing in PBS, the sections were incubated with labelled polymer-HRP for $30 \mathrm{~min}$. The sections were washing in PBS and color was developed with DAB substrate kits.

Combined in situ hybridization and immunohistochemistry. Brain sections were prepared from 11-week-old male C57BL/6 mice, as described above for the doublestaining immunohistochemistry. In situ hybridization of $\beta$-Klotho $(K l b)$ was performed as follows. Sections were washed in $0.1 \mathrm{M}$ phosphate buffer ( $\mathrm{pH} 7.0$ ), then treated with acetylation buffer ( $\mathrm{pH} 8.0$ ) for $10 \mathrm{~min}$. Next, the sections were 
hybridized with $0.5 \mathrm{mg} / \mathrm{ml}$ digoxigenin-labelled sense and antisense RNA probes specific for the Klb sequence (probe sequences are listed in Supplementary Table 2). The sections and antibodies were incubated in diluted hybridization buffer $(50 \%$ [v/v] formamide, $20 \times$ SSC, $10 \%$ blocking reagent, $2 \%$ N-lauroylsarcosine [NLS] and $10 \%$ sodium dodecyl sulphate) for $16-24 \mathrm{~h}$ at $60^{\circ} \mathrm{C}$. Hybridized sections were washed twice at $60^{\circ} \mathrm{C}$ for $20 \mathrm{~min}$ with a mixture of $2 \times \mathrm{SSC}, 50 \%(\mathrm{v} / \mathrm{v})$ formamide, and $0.1 \%$ NLS. Then, sections were washed twice at $37^{\circ} \mathrm{C}$ for $20 \mathrm{~min}$ with a mixture of $2 \times$ SSC and $0.1 \%$ NLS, followed by two more washes at $37^{\circ} \mathrm{C}$ for 20 min with a mixture of $0.2 \times$ SSC and $0.1 \%$ NLS. Subsequently, the sections were incubated for $5 \mathrm{~h}$ at $22-25^{\circ} \mathrm{C}$ with alkaline phosphatase-conjugated anti-digoxigenin antibody (1093274, Roche, Indianapolis, IN, USA), which was diluted at 1:1000 with $1 \%$ blocking reagent. Incubated sections were washed 3 times for 15 min with a mixture of $0.1 \mathrm{M}$ Tris- $\mathrm{HCl}(\mathrm{pH} 7.5), 0.15 \mathrm{M} \mathrm{NaCl}$ and $0.05 \%$ Tween 20. Then, sections were incubated for $16-24 \mathrm{~h}$ at $37^{\circ} \mathrm{C}$ with NBT/BCIP stock solution (Roche), diluted at 1:50 with a mixture of $0.1 \mathrm{M}$ Tris- $\mathrm{HCl}(\mathrm{pH} 7.5), 0.15 \mathrm{M}$ $\mathrm{NaCl}$ and $0.05 \%$ Tween 20 . After two washes with a mixture of PBS and $1 \mathrm{mM}$ EDTA for $5 \mathrm{~min}$, the sections were washed for $24 \mathrm{~h}$ at $37^{\circ} \mathrm{C}$ with a mixture of $0.1 \mathrm{M}$ Tris- $\mathrm{HCl}(\mathrm{pH} 7.5), 0.15 \mathrm{M} \mathrm{NaCl}$ and $0.05 \%$ Tween 20 . A PBS wash followed, then treatment with $0.3 \% \mathrm{H}_{2} \mathrm{O}_{2}$ diluted in PBS for $15 \mathrm{~min}$. The sections were then blocked with $1 \%$ bovine serum albumin and $2 \%$ normal goat serum diluted in PBS with $0.25 \%$ TritonX-100 for $30 \mathrm{~min}$. The sections were incubated overnight with rabbit anti-Oxt antibodies (1:1200) diluted in blocking solution. After being washed in PBS, the sections were incubated with biotinylated goat anti-rabbit IgG $(1: 400)$ for $40 \mathrm{~min}$, then incubated with $\mathrm{ABC}$ reagent for $40 \mathrm{~min}$. The sections were washed three times in PBS, and colour was developed with a diaminobenzidine solution $\left(0.2 \mathrm{~g} / \mathrm{l}\right.$ diaminobenzidine and $0.006 \% \mathrm{H}_{2} \mathrm{O}_{2}$ in PBS).

Single-neuron cytosolic $\mathrm{Ca}^{2+}$ concentration measurement. Single neurons were prepared according to procedures reported previously $y^{68}$ with slight modifications. Briefly, single neurons were prepared from 5-week-old male C57BL/6 mice. The dissected tissues were washed with $10 \mathrm{mM}$ HEPES-buffered Krebs-Ringer bicarbonate buffer (HKRB) containing $10 \mathrm{mM}$ glucose. They were then incubated in HKRB supplemented with $20 \mathrm{U} / \mathrm{ml}$ papain (P4762, Sigma Aldrich), $0.015 \mathrm{mg} / \mathrm{ml}$ DNase II (D-4138, Sigma Aldrich) and $0.75 \mathrm{mg} / \mathrm{ml}$ bovine serum albumin (A2153, Sigma Aldrich) for $16 \mathrm{~min}$ at $36^{\circ} \mathrm{C}$. This incubation was followed by gentle mechanical trituration. Then, the cell suspension was centrifuged at $100 \times g$ for $5 \mathrm{~min}$. The pellet was resuspended in HKRB and distributed onto coverslips.

Cytosolic $\mathrm{Ca}^{2+}$ concentration $\left(\left[\mathrm{Ca}^{2+}\right]_{\mathrm{i}}\right)$ was measured by ratiometric fura- 2 fluorescence imaging. Briefly, after incubation with $2 \mu \mathrm{M}$ fura-2/AM (F016, Dojindo, Kumamoto, Japan) for $40 \mathrm{~min}$, the cells were mounted in a chamber and superfused with HKRB at $1 \mathrm{ml} / \mathrm{min}$ at $33^{\circ} \mathrm{C}$. Moreover, mFGF21 $(50 \mathrm{ng} / \mathrm{ml})$ was administered in the superfusion solution, and $\left[\mathrm{Ca}^{2+}\right]_{\mathrm{i}}$ was measured for $5 \mathrm{~min}$ after addition of mFGF21. Fluorescence images from excitation at 340 and $380 \mathrm{~nm}$ were detected every $10 \mathrm{~s}$ with a cooled charge-coupled device camera (ORCA-R2 C10600, Hamamatsu Photonics, Shizuoka, Japan), and the ratio image was produced by an Aquacosmos (Hamamatsu Photonics)

The single neurons subjected to $\left[\mathrm{Ca}^{2+}\right]_{\mathrm{i}}$ measurements were subsequently immunostained for Oxt using anti-Oxt antibodies. Briefly, the neurons were postfixed in $4 \%$ PFA solution at $4{ }^{\circ} \mathrm{C}$ overnight. Rabbit anti-Oxt antibodies (1:1000) were used as primary antibodies, and Alexa Fluor 594 Donkey Anti-Rabbit IgG (1:400; A-11055, Thermo Fisher Scientific) was used as secondary antibody, respectively. Fluorescence images were acquired using an Eclipse TE2000-U (Nikon, Tokyo, Japan).

Effects of mFGF21 on hypothalamic gene expression. Eleven-week-old male C57BL/6 mice were injected IP with mFGF21 $(1 \mathrm{mg} / \mathrm{kg}$ ) or water (vehicle) $(n=4$ per group). At $30 \mathrm{~min}$ after the injection, mice were killed and the hypothalamus was harvested for gene expression analyses.

Cell culture. Mouse embryonic hypothalamic N41 cells (Cosmo Bio, Tokyo, Japan) were maintained in high-glucose DMEM (Thermo Fisher Scientific) supplemented with $10 \%$ foetal bovine serum (Thermo Fisher Scientific).

\footnotetext{
Adenoviral and lentiviral infections. N41 cells were plated for $24 \mathrm{~h}$ before infections. In the Sirt1 overexpression experiments, N41 cells were infected with an adenovirus that carried a wild-type Sirt1 or GFP (green fluorescent protein; vector control). Infections were performed at a multiplicity of infection (MOI) of 100 to 600 infectious units per cell, and the infections were carried out in culture medium for $24 \mathrm{~h}$ at $37^{\circ} \mathrm{C}$. In the Sirt1 and Nrf2 knockdown experiments, N41 cells were infected with fresh lentivirus that carried one of two Sirt1 short hairpin RNAs (TRCN0000306512 or TRCN0000326966, Sigma Aldrich) or the Nrf2 short hairpin RNA (TRCN0000012132, Sigma Aldrich), respectively. Infections were carried out in DMEM containing $8 \mu \mathrm{g} / \mathrm{ml}$ polybrene for $24 \mathrm{~h}$. Then, cells were cultured for an additional $72 \mathrm{~h}$. Infected cells were selected based on puromycin resistance $(0.5 \mu \mathrm{g} /$ $\mathrm{ml} ; 7$ days). After selection, virus-infected cells were seeded in 24-well plates with culture medium at a density of $1 \times 10^{5}$ cells/well, incubated for $24 \mathrm{~h}$, then subjected to serum starvation for $24 \mathrm{~h}$ before testing the effect of mFGF21 treatment to avoid the influence of various hormones contained in serum.
}

mFGF21, DEM and MAPK inhibitor treatments. N41 cells were seeded in 24-well plates with culture medium at a density of $1 \times 10^{5}$ cells/well and incubated for $24 \mathrm{~h}$ followed by serum starvation for $24 \mathrm{~h}$. Then, cells were untreated or treated with either $100 \mathrm{nM}$ mFGF21 or $100 \mu \mathrm{M}$ DEM (NRF2 activator; Wako) for $0,2,4$ or $8 \mathrm{~h}$. In an additional experiment, the cells were treated for $8 \mathrm{~h}$ with different concentrations of mFGF21 $(0,25,50$ or $100 \mathrm{nM})$ or $\operatorname{DEM}(0,25,50$ or $100 \mu \mathrm{M})(n=3$ per group).

In kinase inhibitor experiments, N41 cells were untreated or treated with either ERK inhibitor (10 $\mu \mathrm{M}$ U0126; Sigma Aldrich) or PI3K inhibitor $(10 \mu \mathrm{M}$ wortmannin; Sigma Aldrich) for $30 \mathrm{~min}$. Then, cells were further incubated for $8 \mathrm{~h}$ with or without addition of $100 \mathrm{nM}$ mFGF21 ( $n=4$ per group).

Quantitative PCR analysis. Total RNA was isolated from the dissected hypothalamus or from N41 cells with RNAiso Plus (Takara Bio, Kyoto, Japan). The isolated RNA was reverse transcribed to CDNA with the Improm II Reverse Transcription System (Promega, Fitchburg, WI, USA). The generated cDNA samples $(1 \mu \mathrm{g})$ were subjected to real-time PCR with the Light Cycler system and Light Cycler 480 SYBR Green I (Roche). The target mRNA levels were expressed relative to the levels of mouse $\beta$-actin. The primer sequences are listed in Supplementary Table 1.

Immunoblot analysis. Total cell lysates and nuclear lysates were prepared using lysis buffer supplemented with a Complete Mini Protease Inhibitor Cocktail tablet (Roche), $100 \mu \mathrm{M}$ MG132 (Sigma) and $0.25 \mathrm{mg} / \mathrm{ml}$ ubiquitin aldehyde (Peptide Institute, Osaka, Japan). To prepare cytoplasmic and nuclear lysates, ice-cold cytoplasmic lysis buffer (5 mM PIPES (KOH) pH 8.0, $65 \mathrm{mM} \mathrm{KCl}$ and $0.5 \% \mathrm{NP} 40$ ) was added and the supernatants after centrifugation recovered as cytoplasmic lysates. Remaining nuclear pellets were mixed with the lysis buffer to make nuclear lysates. The lysates were resolved with polyacrylamide gel electrophoresis, then the separated proteins on the gel were transferred to nitrocellulose membranes. The membranes were probed with primary antibodies against SIRT1 (1:1000; \#07-131, Millipore), ERK1/2 (1:1000; \#9102, Cell Signaling Technology, Danvers, MA, USA), phospho-ERK1/2 (1:1000; \#4376, Cell Signaling Technology), AKT (1:1000; \#9272, Cell Signaling Technology), phospho-AKT (1:1000; \#9271, Cell Signaling Technology), NRF2 (1:1000; \#ab31163, Abcam, Cambridge, UK), $\alpha$-tubulin (1:500 \#sc-5286, Santa Cruz Biotechnology, Dallas, TX, USA) and B23 (1:200; \#sc-6013, Santa Cruz Biotechnology). Then, membranes were incubated with corresponding horseradish peroxidase-conjugated secondary antibodies. Immunoreactive proteins were assessed with a LAS-4000 Image analyser (Fuji Film, Tokyo, Japan) and densitometry with ImageJ software (US National Institutes of Health).

Luciferase assays. For luciferase reporter assays, putative promoter regions of the Oxt gene were amplified from genomic DNA and cloned upstream of the firefly luciferase gene in the pGL3 plasmid (Promega). We cloned different-sized fragments of DNA upstream of the wild-type Oxt open reading frame (-5000, -3090 , $-2992,-2665,-2340,-2000,-1400$ and $-175 \mathrm{bp}$ ) into a pGL3 basic vector. To pinpoint the promoter, we mutated all the base pairs in the antioxidant-response element (ARE; 5'-TGACTCA-3' $\rightarrow$ ATCTGAC) site, at position -2273 to -2266 . This fragment was also cloned into the pGL3 vector. We performed transfections of the pGL3-Oxt promoter or the pGL3-mutated Oxt promoter into cultured N41 cells with Amaxa nucleofection (Lonza, Basel, Switzerland), according to the manufacturer's instructions. The cells $\left(1 \times 10^{5}\right.$ cells/well $)$ were seeded in 24 -well plates before transfections. Firefly luciferase and Renilla reniformis luciferase activities were measured with the Dual Luciferase Reporter assay system (Promega), according to the manufacturer's instructions. Firefly luciferase activities were always normalized to Renilla reniformis activities ( $n=3$ per group).

Chromatin immunoprecipitation assay. N41 cells were transfected using the Lipofectamine 3000 reagent (Thermo Fisher Scientific) according to the manufacturer's instructions. Where indicated, expression plasmids encoding HA-tagged NRF2, FLAG-tagged SIRT1 or pCMV5 (empty) were cotransfected. Chromatin was extracted from N41 cells transfected with plasmids using the Chromatin Extraction Kit (ab117152; Abcam) ( $n=3$ per group). The lysates then were sonicated to shear the chromatin to 500 and $1000 \mathrm{bp}$, keeping the samples ice cold. Immunoprecipitation was performed overnight at $4{ }^{\circ} \mathrm{C}$ with specific antibodies. After immunoprecipitation, DNA was heated at $65^{\circ} \mathrm{C}$ overnight to reverse formaldehyde crosslinking. DNA fragments then were purified with a QIAquick Gel Extraction Kit (Qiagen, Hilden, Germany). Specific antibodies against the HA tag (3F10; Roche) were used for the immunoprecipitation. Normal mouse IgG antibody (sc-2025; Santa Cruz Biotechnology) served as a negative control. Precipitated DNA fragments were analysed by PCR amplification using primers directed against the ARE region in the Oxt promoter.

FGF21 level measurements. Eleven-week old, male WT, OE-OS and OS-KO mice fed NC ad libitum ( $n=5$ per group) were killed, blood was collected and serum was prepared. Eleven-week-old, male OS-KO mice (control, $n=6$; KO, $n=6$ ) and OS-OE mice (control, $n=6$; OE, $n=7$ ) were fasted for $2 \mathrm{~h}$, then they received a sucrose gavage at $2 \mathrm{~g} / \mathrm{kg}$. Sucrose-gavaged mice were killed after $1 \mathrm{~h}$, blood was collected and serum was prepared. The level of mouse FGF21 proteins in each 
serum sample was determined with the Fibroblast Growth Factor 21 Mouse/Rat ELISA kit (BioVendor) according to the manufacturer's instructions.

Leptin level measurements. Eleven-week-old, male WT, OE-OS and OS-KO mice fed NC ad libitum ( $n=8$ per group) were killed, blood was collected and serum was prepared. The level of mouse leptin proteins in each serum sample was determined with the Mouse Leptin ELISA Kit (ab199082; Abcam) according to the manufacturer's instructions.

NAD ${ }^{+}$measurements. We performed quantification of hypothalamic $\mathrm{NAD}^{+}$ levels in brain samples from 11-week-old male C57BL/6 mice, as previously described $^{10}(n=12$ in the Fed group, and $n=13$ in the Fasted group).

Oxt release measurements. The measurements of Oxt release from ex vivo neurons of PVH nuclei from 11-week-old male C57BL/6 mice were performed as previously described ${ }^{69}$.

Statistical analysis. The data were analysed with the Student's $t$-test and Tukey's multiple comparison test following one-way analysis of variance with SPSS 21.0J software. The data are expressed as the mean \pm standard error (SE). All results with $p$ values $<0.05$ were considered statistically significant.

\section{Data availability}

The data that support the findings of this study are available from the corresponding authors upon reasonable request.

Received: 19 December 2017 Accepted: 12 October 2018

Published online: 02 November 2018

\section{References}

1. GBD2015 Risk Factors Collaborators. Global, regional, and national comparative risk assessment of 79 behavioural, environmental and occupational, and metabolic risks or clusters of risks, 1990-2015: a systematic analysis for the Global Burden of Disease Study 2015. Lancet 388, 1659-1724 (2016).

2. Dehghan, M. et al. Associations of fats and carbohydrate intake with cardiovascular disease and mortality in 18 countries from five continents (PURE): a prospective cohort study. Lancet 390, 2050-2062 (2017).

3. Hall, K. D. et al. Energy expenditure and body composition changes after an isocaloric ketogenic diet in overweight and obese men. Am. J. Clin. Nutr. 104, 324-333 (2016).

4. Hall, K. D. et al. Calorie for calorie, dietary fat restriction results in more body fat loss than carbohydrate restriction in people with obesity. Cell Metab. 22, 427-436 (2015).

5. Tay, J. et al. Comparison of low- and high-carbohydrate diets for type 2 diabetes management: a randomized trial. Am. J. Clin. Nutr. 102, 780-790 (2015).

6. Koegler, F. H., Schaffhauser, A. O., Mynatt, R. L., York, D. A. \& Bray, G. A. Macronutrient diet intake of the lethal yellow agouti (Ay/a) mouse. Physiol. Behav. 67, 809-812 (1999).

7. Tung, Y. C., Rimmington, D., O’Rahilly, S. \& Coll, A. P. Pro-opiomelanocortin modulates the thermogenic and physical activity responses to high-fat feeding and markedly influences dietary fat preference. Endocrinology 148, 5331-5338 (2007).

8. van der Klaauw, A. A. et al. Divergent effects of central melanocortin signalling on fat and sucrose preference in humans. Nat. Commun. 7, 13055 (2016).

9. Sasaki, T. Neural and molecular mechanisms involved in controlling the quality of feeding behavior: diet selection and feeding patterns. Nutrients $\mathbf{9}$, E1151 (2017).

10. Sasaki, T. et al. Hypothalamic SIRT1 prevents age-associated weight gain by improving leptin sensitivity in mice. Diabetologia 57, 819-831 (2014).

11. Sasaki T. Age-associated weight gain, leptin, and SIRT1: A possible role for hypothalamic SIRT1 in the prevention of weight gain and aging through modulation of leptin sensitivity. Front Endocrinol. 6, 109 (2015).

12. Anderson, R. M. \& Weindruch, R. Metabolic reprogramming, caloric restriction and aging. Trends Endocrinol. Metab. 21, 134-141 (2010).

13. Moussaieff, A. et al. Glycolysis-mediated changes in acetyl-CoA and histone acetylation control the early differentiation of embryonic stem cells. Cell Metab. 21, 392-402 (2015).

14. DeBerardinis, R. J., Lum, J. J., Hatzivassiliou, G. \& Thompson, C. B. The biology of cancer: metabolic reprogramming fuels cell growth and proliferation. Cell Metab. 7, 11-20 (2008).
15. Muramatsu, K. et al. Neuron-specific recombination by Cre recombinase inserted into the murine tau locus. Biochem. Biophys. Res. Commun. 370, 419-423 (2008).

16. Cheng, H. L. et al. Developmental defects and p53 hyperacetylation in Sir2 homolog (SIRT1)-deficient mice. Proc. Natl Acad. Sci. USA 100, 10794-10799 (2003).

17. Motta, M. C. et al. Mammalian SIRT1 represses forkhead transcription factors Cell 116, 551-563 (2004).

18. Luo, J. et al. Negative control of p53 by Sir2alpha promotes cell survival under stress. Cell 107, 137-148 (2001).

19. Vaziri, H. et al. hSIR2(SIRT1) functions as an NAD-dependent p53 deacetylase. Cell 107, 149-159 (2001)

20. Yeung, F. et al. Modulation of NF-kappaB-dependent transcription and cell survival by the SIRT1 deacetylase. EMBO J. 23, 2369-2380 (2004).

21. Alsio, J., Olszewski, P. K., Levine, A. S. \& Schioth, H. B. Feed-forward mechanisms: addiction-like behavioral and molecular adaptations in overeating. Front. Neuroendocrinol. 33, 127-139 (2012).

22. Klockars, A., Levine, A. S. \& Olszewski, P. K. Central oxytocin and food intake: focus on macronutrient-driven reward. Front Endocrinol. 6, 65 (2015).

23. Amico, J. A., Vollmer, R. R., Cai, H. M., Miedlar, J. A. \& Rinaman, L. Enhanced initial and sustained intake of sucrose solution in mice with an oxytocin gene deletion. Am. J. Physiol. Regul. Integr. Comp. Physiol. 289, R1798-1806 (2005)

24. Billings, L. B., Spero, J. A., Vollmer, R. R. \& Amico, J. A. Oxytocin null mice ingest enhanced amounts of sweet solutions during light and dark cycles and during repeated shaker stress. Behav. Brain. Res. 171, 134-141 (2006).

25. Miedlar, J. A., Rinaman, L., Vollmer, R. R. \& Amico, J. A. Oxytocin gene deletion mice overconsume palatable sucrose solution but not palatable lipid emulsions. Am. J. Physiol. Regul. Integr. Comp. Physiol. 293, R1063-1068 (2007).

26. Sclafani, A., Rinaman, L., Vollmer, R. R. \& Amico, J. A. Oxytocin knockout mice demonstrate enhanced intake of sweet and nonsweet carbohydrate solutions. Am. J. Physiol. Regul. Integr. Comp. Physiol. 292, R1828-1833 (2007).

27. Olszewski, P. K. et al. Molecular, immunohistochemical, and pharmacological evidence of oxytocin's role as inhibitor of carbohydrate but not fat intake. Endocrinology 151, 4736-4744 (2010).

28. $\mathrm{Wu}, \mathrm{Z}$. et al. An obligate role of oxytocin neurons in diet induced energy expenditure. PLoS ONE 7, e45167 (2012).

29. Gomez, J. L. et al. Chemogenetics revealed: DREADD occupancy and activation via converted clozapine. Science 357, 503-507 (2017)

30. Pepino, M. Y. Metabolic effects of non-nutritive sweeteners. Physiol. Behav. 152, 450-455 (2015).

31. Burke, M. V. \& Small, D. M. Physiological mechanisms by which nonnutritive sweeteners may impact body weight and metabolism. Physiol. Behav. 152, 381-388 (2015).

32. von Holstein-Rathlou, S. et al. FGF21 mediates endocrine control of simple sugar intake and sweet taste preference by the liver. Cell Metab. 23, 335-343 (2016).

33. Soberg, S. et al. FGF21 is a sugar-induced hormone associated with sweet intake and preference in humans. Cell Metab. 25, 1045-1053 (2017).

34. Talukdar, S. et al. FGF21 regulates sweet and alcohol preference. Cell Metab. 23, 344-349 (2016)

35. Grinevich, V., Desarmenien, M. G., Chini, B., Tauber, M. \& Muscatelli, F. Ontogenesis of oxytocin pathways in the mammalian brain: late maturation and psychosocial disorders. Front. Neuroanat. 8, 164 (2015).

36. Hume, C., Sabatier, N. \& Menzies, J. High-sugar, but not high-fat, food activates supraoptic nucleus neurons in the male rat. Endocrinology 158 , 2200-2211 (2017).

37. Fisher, F. M. \& Maratos-Flier, E. Understanding the physiology of FGF21. Annu. Rev. Physiol. 78, 223-241 (2016).

38. Ludwig, M. \& Leng, G. Dendritic peptide release and peptide-dependent behaviours. Nat. Rev. Neurosci. 7, 126-136 (2006).

39. Kimura, M. et al. Molecular basis distinguishing the DNA binding profile of Nrf2-Maf heterodimer from that of Maf homodimer. J. Biol. Chem. 282, 33681-33690 (2007).

40. Yu, Y. et al. Fibroblast growth factor (FGF21) protects mouse liver against Dgalactose-induced oxidative stress and apoptosis via activating Nrf2 and PI3K/ Akt pathways. Mol. Cell Biochem. 403, 287-299 (2015).

41. Chen, D. et al. Tissue-specific regulation of SIRT1 by calorie restriction. Genes Dev. 22, 1753-1757 (2008).

42. Sasaki, T. et al. Induction of hypothalamic Sirt1 leads to cessation of feeding via agouti-related peptide. Endocrinology 151, 2556-2566 (2010).

43. Tan, B. K. et al. Fibroblast growth factor 21 (FGF21) in human cerebrospinal fluid: relationship with plasma FGF21 and body adiposity. Diabetes 60 , 2758-2762 (2011).

44. Fisher, F. M. et al. Obesity is a fibroblast growth factor 21 (FGF21)-resistant state. Diabetes 59, 2781-2789 (2010). 
45. Gallego-Escuredo, J. M. et al. Opposite alterations in FGF21 and FGF19 levels and disturbed expression of the receptor machinery for endocrine FGFs in obese patients. Int. J. Obes. 39, 121-129 (2015).

46. Li, Y. et al. Hepatic SIRT1 attenuates hepatic steatosis and controls energy balance in mice by inducing fibroblast growth factor 21. Gastroenterology 146, 539-549 (2014).

47. Qian, W. et al. Decreased circulating levels of oxytocin in obesity and newly diagnosed type 2 diabetic patients. J. Clin. Endocrinol. Metab. 99, 4683-4689 (2014).

48. Lawson, E. A. et al. Oxytocin reduces caloric intake in men. Obesity 23, 950-956 (2015).

49. Heinrichs, M., Baumgartner, T., Kirschbaum, C. \& Ehlert, U. Social support and oxytocin interact to suppress cortisol and subjective responses to psychosocial stress. Biol. Psychiatry 54, 1389-1398 (2003).

50. Zhang, Y. et al. The starvation hormone, fibroblast growth factor-21, extends lifespan in mice. eLife 1, e00065 (2012).

51. Bishop, N. A. \& Guarente, L. Two neurons mediate diet-restriction-induced longevity in C. elegans. Nature 447, 545-549 (2007).

52. Sykiotis, G. P. \& Bohmann, D. Keap1/Nrf2 signaling regulates oxidative stress tolerance and lifespan in Drosophila. Dev. Cell 14, 76-85 (2008).

53. Lewis, K. N. et al. Regulation of Nrf2 signaling and longevity in naturally longlived rodents. Proc. Natl Acad. Sci. USA 112, 3722-3727 (2015).

54. Brunet, A. et al. Stress-dependent regulation of FOXO transcription factors by the SIRT1 deacetylase. Science 303, 2011-2015 (2004).

55. Westerheide, S. D., Anckar, J., Stevens, S. M. Jr., Sistonen, L. \& Morimoto, R. I. Stress-inducible regulation of heat shock factor 1 by the deacetylase SIRT1. Science 323, 1063-1066 (2009).

56. Herisson F. M. et al. Oxytocin acting in the nucleus accumbens core decreases food intake. J Neuroendocrinol 28, https://doi.org/10.1111/jne.12381s (2016).

57. Mullis, K., Kay, K. \& Williams, D. L. Oxytocin action in the ventral tegmental area affects sucrose intake. Brain Res. 1513, 85-91 (2013).

58. Hung, L. W. et al. Gating of social reward by oxytocin in the ventral tegmental area. Science 357, 1406-1411 (2017).

59. van der Klaauw, A. A. et al. Oxytocin administration suppresses hypothalamic activation in response to visual food cues. Sci. Rep. 7, 4266 (2017).

60. Striepens, N. et al. Oxytocin enhances cognitive control of food craving in women. Hum. Brain Mapp. 37, 4276-4285 (2016).

61. Berthoud, H. R., Bereiter, D. A., Trimble, E. R., Siegel, E. G. \& Jeanrenaud, B. Cephalic phase, reflex insulin secretion neuroanatomical and physiological characterization. Diabetologia 20, 393-401 (1981).

62. Herisson, F. M., Brooks, L. L., Waas, J. R., Levine, A. S. \& Olszewski, P. K Functional relationship between oxytocin and appetite for carbohydrates versus saccharin. Neuroreport 25, 909-914 (2014).

63. Klockars, O. A., Klockars, A., Levine, A. S. \& Olszewski, P. K. Oxytocin administration in the basolateral and central nuclei of amygdala moderately suppresses food intake. Neuroreport 29, 504-510 (2018).

64. Palmnas, M. S. et al. Low-dose aspartame consumption differentially affects gut microbiota-host metabolic interactions in the diet-induced obese rat. PLoS ONE 9, e109841 (2014).

65. Suez, J. et al. Artificial sweeteners induce glucose intolerance by altering the gut microbiota. Nature 514, 181-186 (2014).

66. Buffington, S. A. et al. Microbial reconstitution reverses maternal diet-induced social and synaptic deficits in offspring. Cell 165, 1762-1775 (2016).

67. Inutsuka, A. et al. The integrative role of orexin/hypocretin neurons in nociceptive perception and analgesic regulation. Sci. Rep. 6, 29480 (2016).
68. Kohno, D. et al. Sweet taste receptor serves to activate glucose- and leptinresponsive neurons in the hypothalamic arcuate nucleus and participates in glucose responsiveness. Front. Neurosci. 10, 502 (2016).

69. Takayanagi, Y. et al. Activation of supraoptic oxytocin neurons by secretin facilitates social recognition. Biol. Psychiatry 81, 243-251 (2017).

\section{Acknowledgements}

We thank Dr. Brad Lowell at Harvard Medical School and Dr. David Olson at the University of Michigan for providing the Oxytocin-ires-Cre mice, and Dr. Frederick Alt at Boston Children's Hospital for providing the Sirt1-flox mice, respectively. We thank Dr. Bryan Roth at the University of North Carolina for sharing the DREADD plasmid vectors. We thank Ms. C. Oosawa and S. Hashimoto for technical assistance. We thank the Biosignal Genome Resource Center at Gunma University for use of their facility. This work was supported by JSPS KAKENHI 15K19504 (S.M.), 17H01971 (T. Sasaki) and 17H06050 (T. Sasaki), and grants-in-aid from the Mochida Memorial Foundation, the Koyanagi Foundation, and the Health Sciences Foundation (awarded to T. Sasaki).

\section{Author contributions}

Conceptualization, S.M. and T.Sa.; Methodology, S.M., T.Sa., D.K., A.I., A.Y., T.N. and T. O.; Investigation, S.M., T.Sa., K.Y., H.Y.-H., T.N. and T.O.; Writing-Original Draft, S.M and T.Sa.; Writing-Review and Editing, S.M., T.Sa., D.K., A.I., O.K., T.Su., M.K., A.Y., A.H., T.N., T.O. and T.K.; Funding acquisition, S.M. and T.Sa.; Resources, A.H., T.N. and T.O.; Supervision, T.Sa. and T.K.

\section{Additional information}

Supplementary Information accompanies this paper at https://doi.org/10.1038/s41467 018-07033-z.

Competing interests: The authors declare no competing interests.

Reprints and permission information is available online at http://npg.nature.com/ reprintsandpermissions/

Publisher's note: Springer Nature remains neutral with regard to jurisdictional claims in published maps and institutional affiliations.

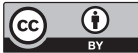

Open Access This article is licensed under a Creative Commons Attribution 4.0 International License, which permits use, sharing, adaptation, distribution and reproduction in any medium or format, as long as you give appropriate credit to the original author(s) and the source, provide a link to the Creative Commons license, and indicate if changes were made. The images or other third party material in this article are included in the article's Creative Commons license, unless indicated otherwise in a credit line to the material. If material is not included in the article's Creative Commons license and your intended use is not permitted by statutory regulation or exceeds the permitted use, you will need to obtain permission directly from the copyright holder. To view a copy of this license, visit http://creativecommons.org/ licenses/by/4.0/

(C) The Author(s) 2018 